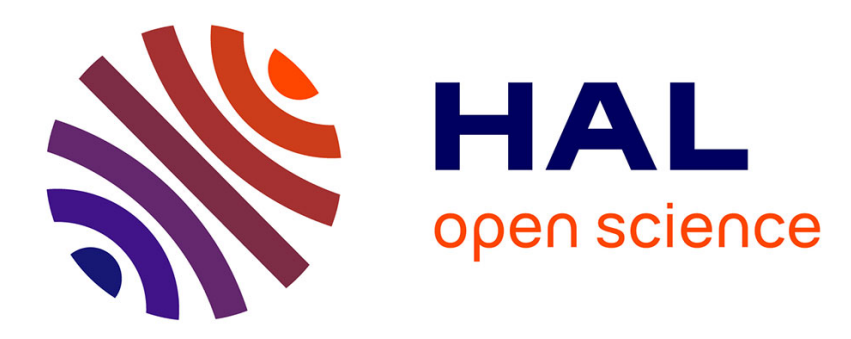

\title{
Progress in rheology and hydrodynamics allowed by NMR or MRI techniques \\ Philippe Coussot
}

\section{To cite this version:}

Philippe Coussot. Progress in rheology and hydrodynamics allowed by NMR or MRI techniques. Experiments in Fluids, 2020, 61 (9), 10.1007/s00348-020-03037-y · hal-03249683

\section{HAL Id: hal-03249683 \\ https://hal-enpc.archives-ouvertes.fr/hal-03249683}

Submitted on 4 Jun 2021

HAL is a multi-disciplinary open access archive for the deposit and dissemination of scientific research documents, whether they are published or not. The documents may come from teaching and research institutions in France or abroad, or from public or private research centers.
L'archive ouverte pluridisciplinaire HAL, est destinée au dépôt et à la diffusion de documents scientifiques de niveau recherche, publiés ou non, émanant des établissements d'enseignement et de recherche français ou étrangers, des laboratoires publics ou privés. 


\title{
Progress in rheology and hydrodynamics allowed by NMR or MRI techniques
}

\author{
P. Coussot \\ Laboratoire Navier, Univ Gustave Eiffel, ENPC, CNRS, 16-20 Bd Newtion, 77420 Champs sur Marne \\ Philippe.coussot@univ-eiffel.fr
}

Note: This paper is dedicated to the memory of my colleague Stéphane Rodts, at the origin so many developments in NMR in Laboratoire Navier

\begin{abstract}
We review the uses of nuclear magnetic resonance techniques for experiments with fluids. More precisely we focus on the progress of knowledge in complex flows, rheology of complex fluids, flow in porous media, colloid transport, fluid transfers in complex porous systems, which have been allowed by NMR techniques. These achievements took advantage of the versatility of NMR, which makes it possible to carry out more original measurements than the basic well-known density imaging (MRI). One may thus rely on non-destructive, non-invasive, local velocimetry, local rheometry, statistical approaches of molecular displacements or velocity, distribution of adsorbed or suspended colloids, evolution of the liquid distribution in different states, fluid transfers during drying or imbibition, etc.
\end{abstract}

\section{Introduction}

In modern science it has become common to observe internal flow characteristics. This is relatively simple for transparent fluids but more complex for non-transparent ones. MRI (Magnetic Resonance lamging) is well-known for its use in medical examination of human body, essentially as a microscopy imaging technique among others, such as echography or X-ray scanning. Actually, at the source of MRI is the physical phenomena which also became a technique, i.e. NMR (Nuclear Magnetic Resonance), which offers a wide field of possibilities of non-destructive measurements inside nontransparent materials. Indeed, with NMR it is possible to excite differently different areas, which allows imaging, but also to get various information on the physical characteristics of the system at a local scale, such as the liquid content and the displacement or velocity of the liquid molecules, through local or statistical measurements. Moreover, since the relaxation of the NMR signal is affected by the interactions of the liquid with its environment (and in particular the surrounding solid phase), NMR also offers the possibility to estimate the liquid state and the pore size or shape in which the liquid lies, or the concentration of suspended elements in a suspension. This made it possible to develop research and make progress in our understanding of various flow types, such as flows of complex fluids or fluid flows in complex structures (such as porous media), so far generally inaccessible to internal information. The objective of the present paper is to review a series of fluid mechanics problems in which one of the various techniques related to NMR or MRI allowed significant progress from the fluid mechanics point of view, generally through quantification of well controlled processes.

In this context, we will start by giving a brief overview of the basic technical elements allowing the reader to understand the principles of the technique and its versatility to measure various flow and material characteristics. Thus, we will not review the most appropriate equipments, materials or NMR procedures (sequences), allowing to get the best measurements for each specific problem, but 
it must be kept in mind that this is in itself a wide scientific field and obtaining relevant and precise measurements requires strong skills and experience. Also note that we will leave apart the specific field of flow measurements for medical applications.

We will consider progress in our understanding of complex flows (i.e. flows with complex boundary conditions) and of the behavior of complex fluids. This naturally leads to the study of flows of suspensions or pasty materials, which are generally non-transparent, transient transport in porous media, transfers between liquid phases in a porous medium, all fields for which it seems that NMR and MRI provided unique internal information making it possible to understand the physical processes. The present information may be completed by reading several other reviews which nevertheless generally insist more on the technical possibilities of NMR or MRI: characterization of porous media by NMR (Barrie 2000), MR velocimetry (Elkins and Alley 2007), adsorption, diffusion and flow (Gladden and Mitchell 2011), mass transfers (Koptyug and Sagdeev 2002), turbulent flows (Sederman et al 2004).

\section{The versatility of Nuclear Magnetic Resonance for measuring flow properties}

The various possibilities of NMR spectroscopy and imaging are described in usual textbooks such as those of Ernst et al (1987) for spectrometry and Callaghan (1991) for imaging and diffusion. Here we just review basic aspects and give a synthetic presentation which allow to understand the specificity of NMR and the possibilities this technique offers in the field of fluid mechanics.

Atomic nuclei are characterized by different states of energy. When they are set in a magnetic field their equilibrium distribution in the corresponding energy levels is displaced. Within the frame of an (approximate) classical mechanics treatment the nuclei may be considered as charged particles exhibiting a magnetic moment (spin). When it is immersed in a magnetic field ( $\left.\mathbf{B}_{0}\right)$ of amplitude $B_{0}$ it may be shown that this spin follows a precession motion, i.e. it turns around the magnetic field direction:

$$
M(t)=M_{0} \exp \left(i \omega_{0} t\right)
$$

in which $\omega_{0}=\gamma B_{0}$ is the Larmor frequency, with $\gamma$ the gyromagnetic ratio of the particle. Since there are in addition energy exchanges due to mutual interactions between material components, the spin progressively tends to align along the direction of the magnetic field. In practice this may be described by simple relaxation processes concerning separately the spin components parallel and perpendicular to the field direction, namely $\mathbf{M}_{/ /}$and $\mathbf{M}_{\perp}$. The corresponding relaxation times are respectively $T_{1}$ and $T_{2}$, and simple relaxation equations may then be used to describe each process. These relaxation times depend on the energy exchanges between the spins and with the surroundings. In particular their values decrease when the liquid is confined in smaller pores.

When some small magnetic field in a different (but constant) direction is repetitively added to this main field, it may ultimately tend to destabilize the spin (which then tilts from the main direction), this is resonance. When this additional field is released the spin more or less rapidly gets back (i.e. it relaxes) towards its equilibrium position. These phenomena may be detected from the electrical current they induce in a coil. Since the magnetic fields acts "at distance" inside the material and induces reversible effects the technique is non-invasive and non-destructive.

If a magnetic field gradient $(\mathbf{G})$ is added to the main field, this one becomes $\mathbf{B}(\mathbf{r})=\mathbf{B}_{0}+\mathbf{G} \cdot \mathbf{r}$, and the spin now precesses at a Larmor frequency $\omega(\mathbf{r})=\gamma B$ which depends on its position $\mathbf{r}$ within the material. Imaging from magnetic resonance is based on the fact that the proton spins precess at different rates as a function of the field value in the different regions they go through, which induces 
a phase shift between spins having followed different paths. Over a short duration $t$ this motion may be expressed in terms of the current position: $\mathbf{r}(t)=\mathbf{r}_{1}+t \mathbf{v}_{1}+o(t)$, in which $\mathbf{r}_{1}=\mathbf{r}(t=0)$ and $\mathbf{v}_{1}=\mathbf{v}(t=0)$ but we will drop the indices in the following. Let us consider a small fluid element in which the velocities of the different elements do not differ significantly during a typical duration $\Delta t$ of the elementary step of a NMR sequence (say several tenths of milliseconds). The total phase shift of the spins in this fluid element is found by integration of the elementary phase shifts resulting from the different magnetic fields to which this element was submitted during its motion. Taking into account in addition the relaxation, the NMR signal of this fluid element finally expresses as (at order $t)$ :

$m=m_{0} \exp \left[i \gamma B_{0} \Delta t+i \mathbf{k} \cdot \mathbf{r}+i \mathbf{p} \cdot \mathbf{v}-t / T_{\text {relax }}(\mathbf{r})+o(t)\right]$

with $\mathbf{k}=\gamma \int_{\Delta t} \mathbf{G}(t) d t$ and $\mathbf{p}=\gamma \int_{\Delta t} t \mathbf{G}(t) d t$.

Most of the versatility of NMR is contained in equation (2). Through $m_{0}(\mathbf{r})$ the signal is proportional to the local spin density, and thus to the mass density of elements containing the resonating spins. Through the first term in the exponential the phase shift varies with the gyromagnetic ratio, and thus with the atom under study, so that different species may be detected by using different resonance frequencies. Through the second term in the exponential the phase shift varies with the proton position; if the other terms remain constant the total NMR signal, i.e. $S=\int m(\mathbf{r}) \mathrm{d} \mathbf{r}$ where the integral is taken over the sample volume imaged, writes

$$
S=\int m_{0}(\mathbf{r}) \exp [i \mathbf{k} \cdot \mathbf{r}] \mathrm{d} \mathbf{r}
$$

We recognize the Fourier Transform of $m_{0}(\mathbf{r})$. Since at each step of any NMR experiment the total signal from the sample is recorded, density imaging will consist to make a series of measurements of $S$ for different values of $\mathbf{k}$ (i.e. different values of the imposed magnetic field gradient) to finally get an image of the density field from the inverse Fourier Transform of $S(\mathbf{k})$. This is standard MRI, that we will often call MR density imaging in the following, to be more precise.

Velocity imaging can be achieved by imposing a history of $\mathbf{G}$ such that $\int_{\Delta t} \mathbf{G}(t) \mathrm{d} t=0$, so that if the other terms are constant phase shift in

$S=\int m_{0}(\mathbf{r}) \exp [i \mathbf{p} \cdot \mathbf{r}] \mathrm{d} \mathbf{r}$

may be used to finally get the velocity field in the sample. This is MR velocimetry.

Note that from a general point of view more detailed information (i.e. better resolution) is obtained from more measurements at different values of the gradients, which is obviously at the price of larger experimental duration. On another side, more information on the motion (such as the acceleration) can be in principle obtained by looking at further orders of development of the position.

At last, the phase shift being sensitive to the spin relaxation the images obtained through the above described techniques may be "weighted" by the relaxation time, if this relaxation time varies from one place to another in the sample. When the physical origin of this relaxation time difference is known this provides an image of the field of this physical characteristics. On another side, it is possible to look directly at the statistical distribution of the different relaxation times existing in the system, by analysing the relaxation process in details, which finally provides a quantification of some liquid transfers at a local scale in the material. 


\section{Complex fluid flows}

In order to check the validity of models or simulations it is useful to measure the velocity field of a flowing fluid. Different ways exist for such measurements: Particle Imaging Velocimetry (PIV) (Raffel et al 1998), Dynamic Light Scattering (Fuller 1995, Salmon et al 2003), Laser Doppler Anemometry (Durst et al 1976), ultrasounds (Gallot et al 2013). These techniques are based on the analysis of image correlation, signal attenuation or scattering. Although some of the above techniques have been used marginally for studying some complex flows or fluids, MRI velocimetry is a unique tool for studying the flow properties of non-transparent materials or concentrated suspensions through complex geometries, as encountered in various industries (concrete, mortars, muds, mining residues, toothpastes, food pastes, paints, etc). Note that such materials are often yield stress fluids (see next section for more details), which flow only when a stress larger than a critical value is applied otherwise they behave as solids; complex flows of such materials generally exhibit original trends (Coussot 2014). MR velocimetry has been used to get information on the flow characteristics of such material type during extrusion, i.e. flow through an orifice of a die, a process widely used for pasty material moulding in industry. This provides a view of the possible dead zones and the shear intensity distribution in the rest of the fluid, in particular at the entrance in the die (Barnes et al 2006, Rabideau et al 2010, Iwamiya et al 1994, Rodts et al 2010. It is also possible to observe the development of more concentrated zones around and before the die entrance (Rabideau et al 2012). The inverse process, i.e. yield stress fluid flow entrance in a larger conduit, could also be described, showing in particular the migration of suspended particles (Jossic et al 2002, Jossic and Magnin 2005, Moraczewski et al 2005). In the case of the expansion flow of a homogeneous (simple) yield stress fluid in a short conduit of larger diameter, a surprising effect of shear-band around the penetrating fluid could be observed (Chevalier et al 2013).
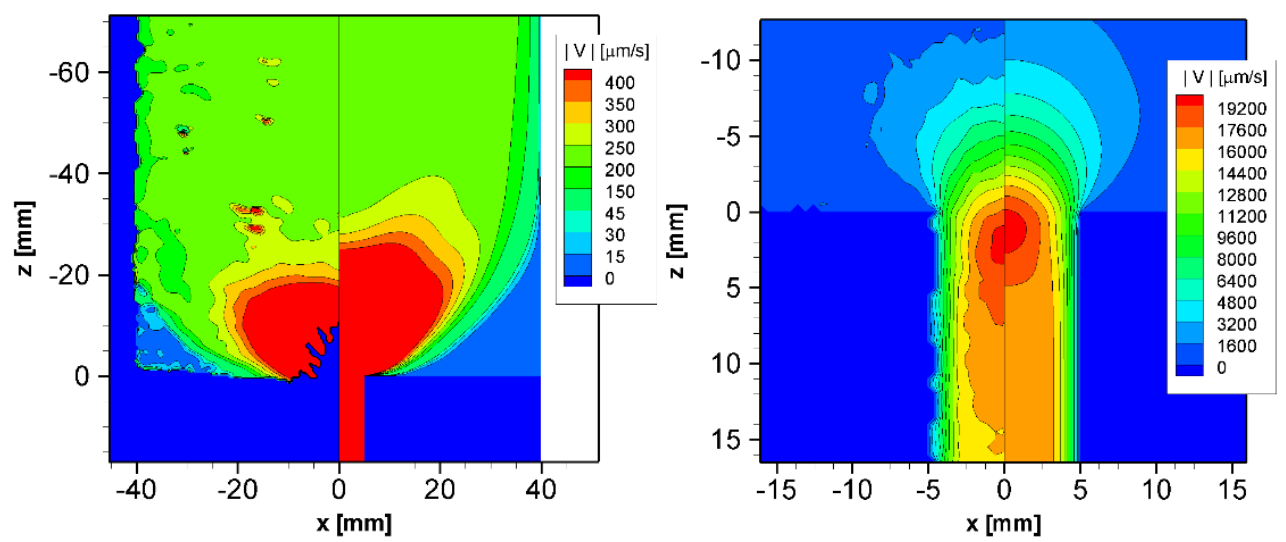

Figure 1: 2D Velocity field in a longitudinal cross-section of the flow of a yield stress fluid (a Carbopol gel) through a conduit ended by a die (contraction ratio: 8): (a) velocity field in the main conduit, (b) zoom in the entrance and die region. The flow is imposed by a piston upward, moving at $200 \mu \mathrm{m} / \mathrm{s}$. MR velocimetry data are shown on the left part of each picture, while the results of simulations are shown on the right. The simulation assumes a simple yield stress fluid behavior with linear elasticity in the solid regime up to yielding. The observed differences might be due to the fact that elastic effects were not properly accounted for in this model. Copyright Figure 14 a,b of Rabideau et al 2010. 
Beyond the observation of flow characteristics allowing some qualitative comparison with simpler fluids in the same geometry, a step further in the analysis of such data can be made by comparing the experimental data with numerical simulations of the flow field (see Figure 1). For these complex fluids (i.e. pastes or concentrated suspensions) this must rely on an appropriate program and the implementation of a relevant tensorial constitutive equation, the latter point being still a matter of intense debate in the field of yield stress fluids (Saramito 2007, Saramito and Wachs 2017, Mendes 2011, Mendes and Thompson 2012, Fraggedakis et al 2016, Bleyer et al 2015, Chaparian and Frigaard 2017, Dimitriou and McKinley 2019). Finally, except in some cases (Zhang et al 2018 a, Varchanis et al 2020) the validity of these constitutive equations is essentially tested from the analysis of simple shear flows. For simple expressions this can be straightforward: this is for example the case of the basic 3D Herschel-Bulkley expression (Coussot 2005) which contains no more parameters than in the simple shear expression; however, as for the others, this tensorial expression has not been proved to apply for more complex flows. More systematic development and comparison of MR velocimetry for such fluid flows with complex boundary conditions could thus constitute an important step forward as it would theoretically allow to determine the form of the constitutive equation and the involved rheological parameters. However a fundamental point has to be taken into account with such fluids in the analysis of the velocity field (Coussot 2014), which contrasts with similar approach for simple fluids: in non-uniform flows, the solid and liquid regions are not established, they can evolve from one point to another, so that some significant deformation in the solid regime can take place in some given region, which must not be confused with flow in the liquid regime. Thus, the analysis of velocity field for such materials even for stationary flows cannot rely on a simple Eulerian description.

Besides, MRI velocimetry has been used in a variety of flow situations, such as the evolution of the composition of a drying sessile droplet (Kind and Thiele 2019), flow in a 3D meandering channel (Wiese et al 2018), turbulent inclined jet in crossflow (Milani et al 2019), concentration distribution during injection in another liquid (Banko et al 2020), flow in a model urban canopy (Shim et al 2019), Taylor-Couette instability (Seymour et al 1999). Remarkably, for the Taylor-Couette change of stability in a coaxial cylinder geometry with a superimposed axial flow, a complete view of the internal characteristics could be obtained (Vallatos et al 2012). The global aspect of the axial displacement and a full characterization of the velocity field in the three directions are shown in Figure 2. The physical processes were then further described from the determination (by NMR) of the conditional probability density for displacement (Vallatos et al 2012).

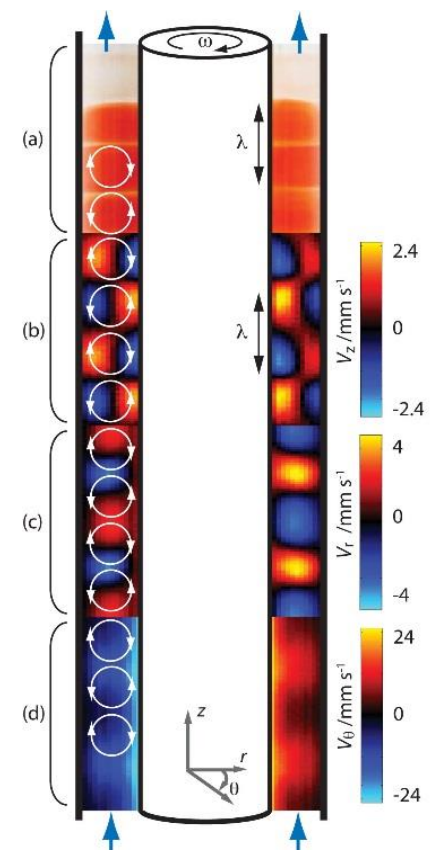


Figure 2: Taylor-Couette change of stability with superimposed axial flow observed by MRI. Different flow characteristics are shown for the same region $(4.75 \times 14 \mathrm{~mm})$ of the system: Density imaging after injection of a ferroin solution from downstream, giving the impression of a progression of the vortices as a plug (a); velocity maps of the flow in the axial (b), radial (c) and azimuthal (d) directions. Copyright Figure 2 of Vallatos et al (2012).

\section{Complex fluids and rheology}

\subsection{Velocimetry of complex fluids}

Rheometry is the set of techniques developed for measuring the constitutive equation of materials. For fluids these techniques rely on the use of boundary conditions allowing to approach as close as possible viscometric flows, i.e. flows for which there exists a referential in which the strain rate tensor exhibits a simple form with only one non-zero term (a diagonal one) (Coleman et al 1966). This term is proportional to the shear rate amplitude $(\dot{\gamma})$ (velocity gradient). The relationship between the corresponding shear stress amplitude $(\tau)$ and the shear rate defines the (intrinsic) constitutive equation of the material in simple shear. It may be very simple, as for Newtonian fluids ( $\tau \propto \dot{\gamma}$ ), or much more complex and in particular depend on flow history, as is the case for yield stress fluids, viscoelastic or thixotropic materials.

Usual rheometers measure macroscopic characteristics of the flow such as the force or the torque exerted along some boundary of the fluid and the relative velocity of boundaries. The corresponding data are generally interpreted by assuming that the material and flow characteristics (typically the shear rate) are homogeneous in the gap between boundaries. This makes it possible to deduce the shear stress (force per unit area of contact between the tool and the material) and the shear rate (relative velocity between tools divided by the gap thickness). For complex fluids this assumption may easily fail due to the non-linearity of the material behavior and/or due to the heterogeneity of its components (e.g. suspensions). It follows a range of effects possibly perturbing the flow, such as migration or sedimentation leading to density heterogeneity, slip at the wall precluding a correct shearing of the material, shear rate heterogeneity or even shear-banding (Coussot 2005). These artefacts induce deviations of the material characteristics (homogeneity) or flow characteristics from the assumptions made for calculations based on macroscopic measurements which cannot take them into account (see Coussot 2005). MRI appears as an ideal means to analyze in details such problems. This was for example done by Ovarlez et al (2011) for edge effects in Couette flow induced by a vane (instead of the inner cylinder) or Zhang et al (2018 b) to measure the wall slip velocity of pastes along smooth surfaces. However, this is concerning the velocity field and the density distribution that most progress has been done (see below). 


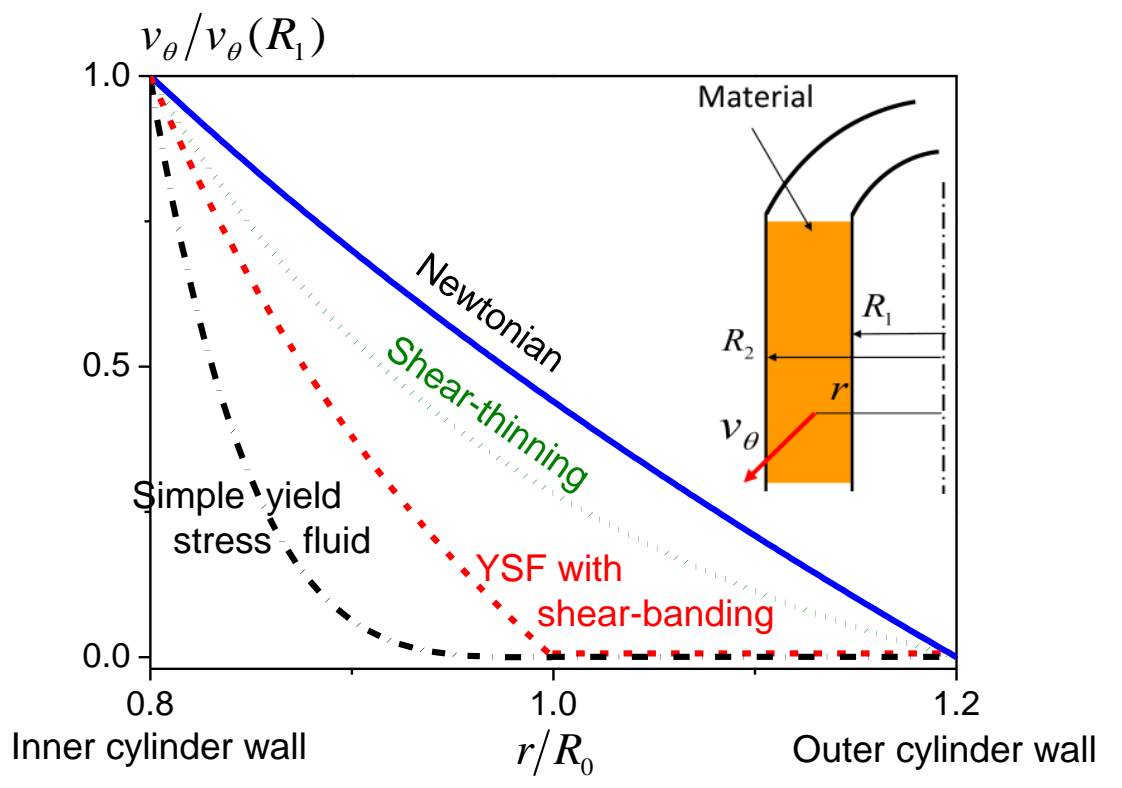

Figure 3: Typical (theoretical) tangential velocity profiles for the flow of different material types in a Couette geometry with inner and outer cylinder radii $R_{1}$ and $R_{2}$. The tangential velocity is rescaled by the maximum velocity (i.e. the velocity along the inner cylinder). The distance from central axis is rescaled by a value $R_{0}=\sqrt{C / 2 \pi h \tau_{c}}$, the distance corresponding to the solid-liquid transition for the yield stress or shear-banding fluids considered here. The rheological models assumed here are: (shear-thinning) power-law model $\left(\tau=k \dot{\gamma}^{n}\right)$ with $n=0.4$, (yield stress fluid) Herschel-Bulkley model ( $\tau=\tau_{c}+k \dot{\gamma}^{n}$ for $\tau>\tau_{c}$ ) with $n=0.4$, and (shear-banding yield stress fluid) truncated power-law model ( $\tau=k \dot{\gamma}^{n}$ for $\tau>\tau_{c}$ ). Note the difference in velocity profile between a simple yield stress fluid and a shear-banding yield stress fluid: the shear rate at the liquid-solid transition for the latter is strictly positive while it tends to zero for the former.

The ideal configuration to observe the non-linearity of the fluid behavior readily appears to be the Couette (coaxial cylinders) system. Indeed, in the absence of inertia effects, if the fluid deformation exhibits a cylindrical symmetry, it may be shown from momentum equation (see Coussot 2005) that the shear stress writes $\tau=C / 2 \pi r^{2} h$, in which $C$ is the applied torque and $h$ the sample height. The resulting variation of the stress with the distance from the system axis is all the stronger as the gap is larger and the material behavior all the more deviates from linearity. This leads to various types of velocity profiles in the gap (see Figure 3). A first approach to quantitatively analyze such data (i.e. velocity profile measurements) in terms of fluid rheological behavior consists to compare the experimental profile with the one expected from the fluid behavior determined through standard (macroscopic) rheometrical measurements (Coussot 2005). This was for example successfully done for polymers and suspensions (Sinton and Chow 1991) and for Xanthan gum (Blythe et al 2015). A similar approach can be carried out from velocity profile data in a capillary geometry, since again in that case, in steady state uniform flow, the stress distribution is given: $\tau=r \partial p / 2 \partial z$, where $\partial p / \partial z$ is the pressure gradient along the capillary axis. The technique was used with Xanthan gums (Haavisto et al 2017), concentrated emulsions (Hollingsworth and Johns 2004, Zhang et al 2018 b) and a few other materials (Seymour et al 1994). 
MRI velocimetry also allowed to directly observe an extreme non-linearity induced by the behavior of some systems in any type of geometry (even the cone and plate one for which the shear stress is almost perfectly uniform). This is shear-banding, which corresponds to a localization of deformation in a specific region with a shear rate anywhere in this region larger than a finite value (see Figure 3 ). The first direct MRI observation of this type concerned micellar solutions (Britton and Callaghan 1997a,b, 1999). This was afterwards confirmed by similar tests in Couette cells (Douglass et al 2008), but later, more sophisticated NMR measurements showed that shear-banding fluctuations (LopezGonzalez et al 2006, Al-kaby et al 2018) and exchanges between coexisting phases (Medronho et al 2011) also occur, in keeping with observations with other techniques (Fardin and Lerouge 2012).

On another side, MR velocimetry made it possible to observe shear-banding in a variety of colloidal suspensions: clay-water suspensions (Coussot et al 2002 a, Raynaud et al 2002), silica suspensions (Moller et al 2008), hard-sphere suspension (Wassenius and Callaghan 2005), colloidal star polymers (Rogers and Callaghan 2009, Holmes et al 2004), Cement pastes (Jarny et al 2005), attractive emulsions (Ragouilliaux et al 2007), drilling fluids (Ragouilliaux et al 2006), waxy crude oils (Mendes et al 2015), wax suspensions (Andrade et al 2020). For these materials, shear-banding was shown to be intimately linked to the thixotropic behavior of the suspension (Ovarlez et al 2009), the material behavior evolving to a liquefied state, beyond a critical stress, and to a solid, restructured state, below this stress (Coussot et al $2002 \mathrm{~b}$ ).

\section{2 "Local rheometry"}

Although the above approach is interesting as it gives unique information about the flow characteristics inside the gap of a rheometer (Couette or capillary), a more powerful approach has progressively developed for analyzing such data, starting as far as we know with the works of Ovarlez et al (2006) and Ragouilliaux et al (2006). It consists to directly associate each local shear stress value (according to the above expressions) to the local shear rate deduced from the slope of the velocity profile through the expression: $\dot{\gamma}=v_{\theta} / r-d v_{\theta} / d r$ (Couette) or $\dot{\gamma}=d v_{z} / d r$ (capillary). Then a plot of the corresponding pairs of shear stress vs shear rate constitutes the rheogram, i.e. the apparent flow curve (stress vs shear rate curve), of the material obtained from local measurements. The whole approach may be considered as local rheometry, as it directly probes the constitutive equation of the material at the scale of the resolution of the MR velocimetry technique. Note however that some additional uncertainty necessarily appears from the fact that the shear rate determination requires a derivative of the velocity profile, a process which enhances uncertainties. Different curves may be obtained from tests at different relative rotation velocities of the cylinders. The overlap of such curves (see Figure 4) then proves the consistency of the constitutive equation obtained in this way, as this indeed means that a single local rheological behavior is obtained for a given shear stress. Local rheometry by MR velocimetry made it possible to determine the effective rheological behavior of Newtonian fluids (Nikolaeva et al 2020), simple yield stress fluids such as Carbopol gels (Coussot et al 2009, Nikolaeva et al 2020) and emulsions (Ovarlez et al 2008). In that case, the consistency of these data with the flow curve determined from standard rheometry could also be checked (see Figure 4). For systems exhibiting shear-banding this technique leads to a truncated flow curve, which predicts that no flow can be obtained at shear rate below a critical, finite, value (see Figure 4). In that case the technique was used for bentonite (clay)-water suspensions (Coussot and Ovarlez 2010, Chernoburova et al 2018) and for wax suspensions (Andrade et al 2020). 


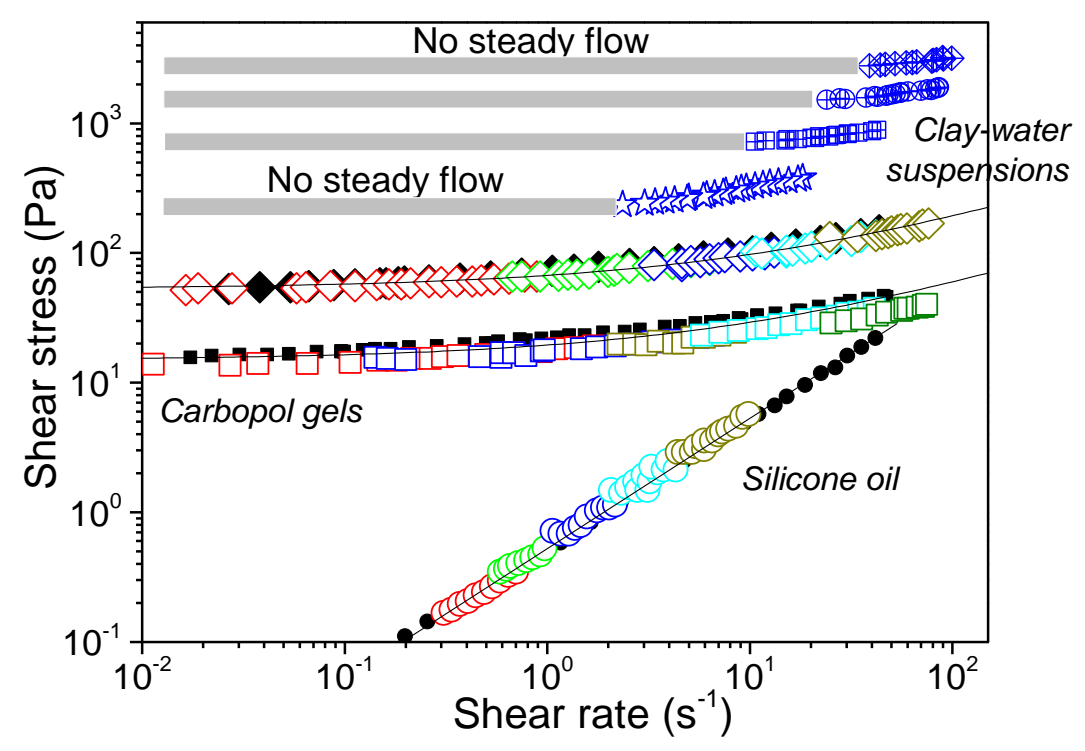

Figure 4: Data from local rheometry by MRI and comparison with data from standard (cone and plate) rheometry (filled symbols): silicone Newtonian oil (circles) (Nikolaeva et al 2020), yield stress Carbopol gels (Nikolaeva et al 2020) (squares and diamonds). The different colors correspond to tests carried out at different rotation velocities. The continuous lines are models fitted to data (Newtonian or Herschel-Bulkley models). The (blue) upper data correspond to bentonite-water suspensions at different concentrations (Coussot and Ovarlez 2010), exhibiting shear-banding, i.e. no steady state flows could be observed for shear rate along the grey range.

Density MRI also made it possible to detect the development of concentration heterogeneities in flowing suspensions. This effect, which was essentially observed in Couette flow (Graham et al 1991, Abbott et al 1991, Chow et al 1994, Corbett et al 1995, Götz et al 2001) and in capillary (Brown et al 2009), is a common shear-induced diffusion which takes the form of a particle concentration increase towards the outer radius in Couette and towards the center in capillary. The concentration profile in steady state could then be compared (Colbourne et al 2018) with some existing model of this phenomenon. Obviously, when such an effect occurs, it precludes a relevant analysis of the constitutive equation on the basis of the simple relationship between the local shear stress and shear rate (see above), since we have a different material (of different composition) at any point in the system. However, the complete information provided by NMR measurements on the local velocity and particle concentration still allows to approach the intrinsic behavior of the material, as shown by Ovarlez et al (2006), which deserves to be detailed here. We now expect the constitutive equation in simple shear to write in the form $\tau(\dot{\gamma}, \phi)$, in which $\dot{\gamma}$ can be determined from MR velocimetry and $\phi$ (the particle concentration) from MR density imaging (see insets of Figure 5). For a model suspension at concentrations close to the maximum packing fraction, the intrinsic viscosity (i.e. $\tau / \dot{\gamma}$ ) could thus be deduced (see Figure 5). This showed that this effective local viscosity is significantly larger than the standard semi-empirical prediction of Krieger and Dougherty (1959) (see Figure 5), which so far could only be compared with experimental data strongly affected by concentration gradient. These observations by MRI also showed that this migration develops very rapidly, typically after one revolution. 


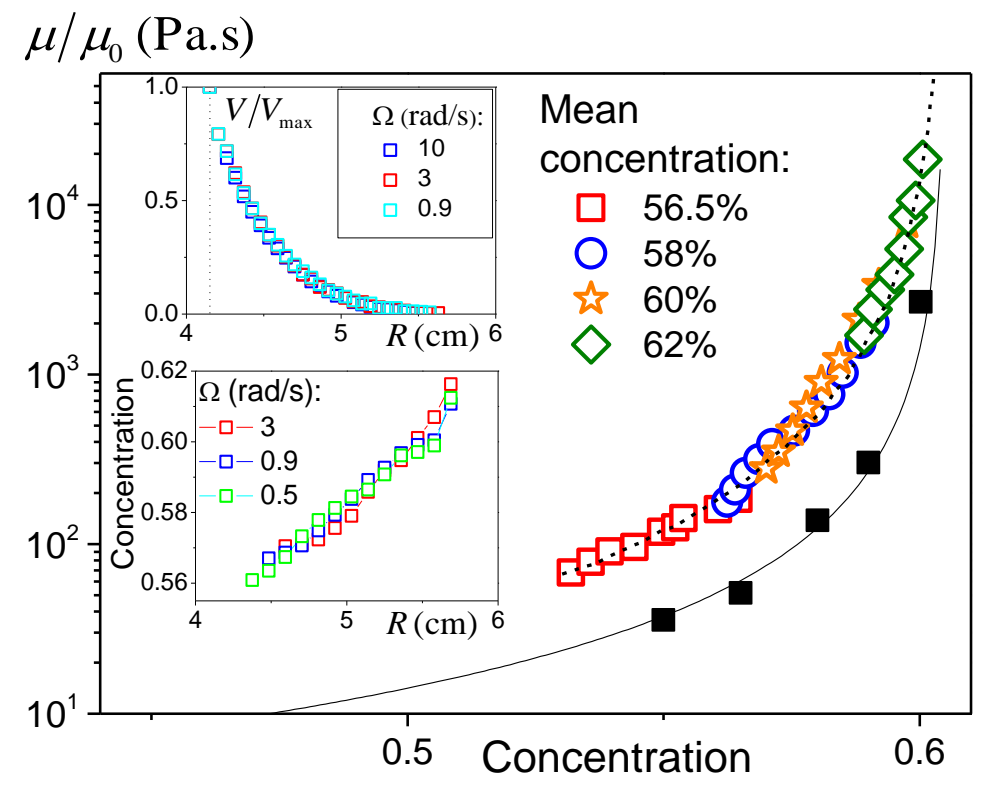

Figure 5: Local viscosity (rescaled by the interstitial liquid viscosity) as a function of concentration for model suspensions of coarse particles in a Newtonian liquid, deduced from local measurements of the velocity (top inset) and the concentration (bottom inset) as a function of the distance from the axis. Note that these data correspond to flow regimes for which it was observed that the torque varies linearly with the rotation velocity. Since the velocity profile does not vary in this range (see top inset), this macroscopic linear behavior ensures that the local behavior is Newtonian and implies that the viscosity is a function of the concentration only. Data shown in the insets here correspond to a mean concentration of $58 \%$. The viscosity data obtained for different mean concentrations and shown in the main graph fall along a master curve, which ensures the consistency of the approach. The continuous line is the Krieger-Dougherty model $\left(\mu / \mu_{0}=\left(1-\phi / \phi_{m}\right)^{-2.5 \phi_{m}}\right)$ fitted to data obtained by standard macroscopic rheometrical measurements (i.e. assuming homogeneous concentration) with $\phi_{m}=60.5 \%$. Data from Ovarlez et al 2006.

So far we focused on the steady state behavior of complex fluids, but such materials also often exhibit a behavior depending on the flow history, this is thixotropy. MR velocimetry can be used to study the thixotropic behavior of fluids. Obviously the steady state flow curve after different times of rest leading to different initial states of structure can be determined. It was thus shown in the case of bentonite suspensions that the apparent flow curve of a typical thixotropic fluid may depend on the preliminary time at rest before imposing some shear: the velocity profile in steady state is then localized in a thinner region for an increasing time at rest (Raynaud et al 2002). When the time evolution of the velocity profile is sufficiently slow with regards to the time needed for MR velocity measurements, it is also possible to observe the evolution of the velocity profile following a step change of the rotation velocity. With the help of the above approach such data may be interpreted in terms of an apparent flow curve evolving in time (Ragouilliaux et al 2006), associated with some evolution of the structure of the material (possibly the number of links between the elementary components). In the absence of more information concerning this structure these apparent flow curves constitute a phenomenological description of the thixotropic behavior. One can hope that in the future it will possible, at least for some systems, to establish some connection between this local apparent viscosity and the relaxation time, the diffusivity or any other property accessible from NMR 
measurements which could provide a physical characterization of the structure. This would make it possible to get a complete picture of the constitutive equation of thixotropic fluids (one of the most complex problem in rheology), in the spirit of the approach described above for concentrated suspensions, i.e. with here the stress being a function of the local shear rate and state of structure.

\section{Flows in porous media}

\subsection{Direct flow measurements}

Porous media are materials which constitute an ideal target for the application of NMR as they are non-transparent but contain liquid dispersed throughout the structure. The basic property of a porous medium being its permeability, i.e. the easiness with which a fluid is able to flow through it, which is in general determined "macroscopically", it is natural to first attempt to use NMR to get information on the internal flow characteristics when a liquid steadily flows through the medium. MR velocimetry was for example used to determine the average velocity distribution in a medium with a transverse permeability gradient (Pavlovskaya et al 2018), allowing to study directly the impact of permeability on the mean flow characteristics and compare it with theoretical predictions or numerical simulations. For porous media, one may nevertheless expect to get more details on the flow by measuring the velocity field at a local scale, i.e. within the pores. However, since the velocity varies in a wide range from the walls (where, in the absence of wall slip, the velocity is equal to zero) to the center of the flow, obtaining sufficient information at first sight means to get a detailed velocity field at the pore scale. Thus, if, as expected for a homogeneous porous medium, the pore scale is much smaller than the sample scale, this requires to have a resolution very much smaller than the sample size, which is hardly accessible with existing equipments.

Another possibility for measuring the velocity field in a porous medium consists to use indexmatched fluid- solid systems and then either track suspended particles moving with the fluid or relying on the Doppler effect (see reviews by Wood et al (2015) or Widerseiner et al (2011)). Nice velocity fields can be obtained in relatively small samples, but the limitations of such techniques as compared to MR velocimetry are as follows: we need to have transparent fluid and porous structure; measurements are more uncertain at increasing depth in the sample, due to a path going through an increasing number of liquid-solid interfaces.

When some satisfactory velocity field has been obtained, this provides a nice insight in the internal flow characteristics, but its analysis remains problematic: the detailed information at the pore scale depends much on the local boundary conditions, which vary from one pore to another in a disordered porous system. As a consequence the velocity field at the pore scale in some pores cannot be considered as providing an information representative of the flow characteristics in the whole system. For example, some heterogeneities (main flow in specific paths) have been observed in such cases (Sederman et al 1998, Götz et al 2002, Ren et al 2005, Lovreglio et al 2018), which are likely specific of the boundary conditions chosen. In particular, Ren et al (2005) showed that heterogeneities and edge effects tend to become negligible for sufficiently large conduit to bead diameter ratios. As for complex flows (see Section 3), a step further in the analysis of such data implies to compare them with the predictions from numerical simulations, as was done by Lovreglio et al 2018 (see Figure 6). If successful, this allows to validate the simulations which may then be used to predict the flow characteristics, and in particular the permeability, of other systems. Other more original uses of direct MR velocimetry inside porous media concern the imaging of the melt front propagation in a phase change material inside a porous medium (Skuntz et al 2018), and the determination of the internal velocity field of gas-liquid flow through porous medium (Sankey et al 2009), using sulfur hexafluoride as gas to detect its motion. 
(a)

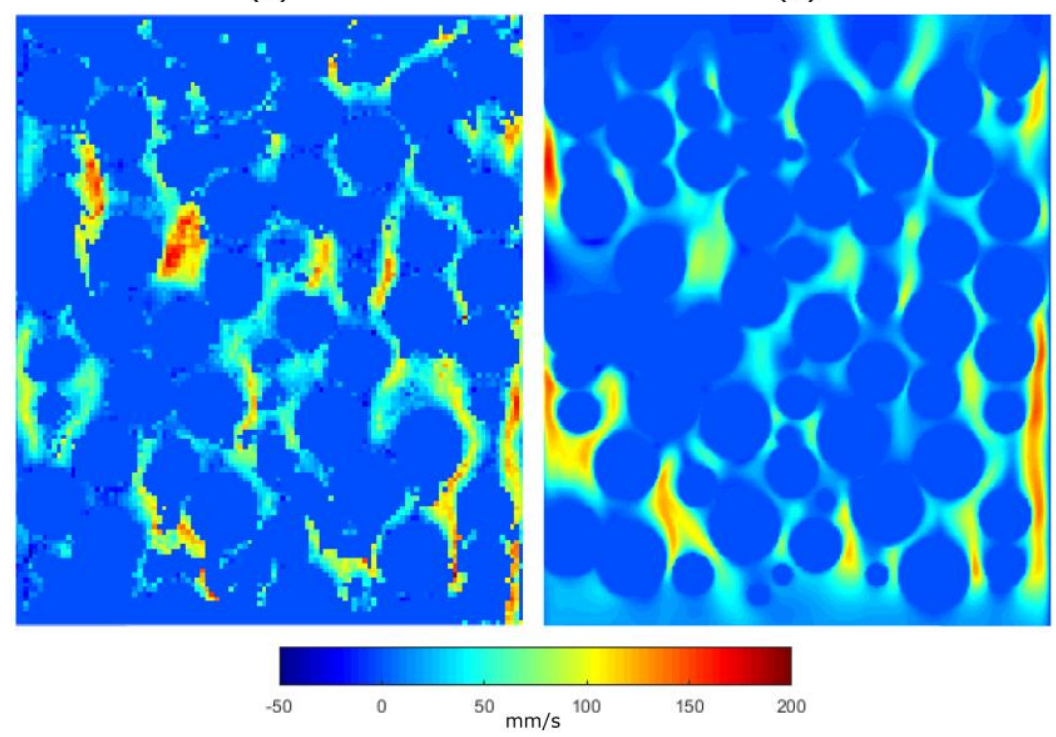

Figure 6: Velocity field for the flow through a packed bed of spheres of diameter $3 \mathrm{~mm}$ : (a) data from MRI, (b) data from numerical simulations. Copyright Figure 10 of Lovreglio et al 2018.

\subsection{Statistical approach of the velocity distribution}

The above approaches remains somewhat onerous for a limited result, while NMR offers more powerful techniques to study flow characteristics in porous media. Actually, the particular nature of porous media made of a large number of pores in which the detailed velocity field varies from one pore to another, suggest to look at the flow characteristics in a different way taking into account the statistical nature of the system. This consists to measure some local physical quantity related to flow and determine the statistical distribution of this property. In that frame, NMR has the great advantage to be a technique which records the signal arising from all the elements at the elementary scale (protons), i.e. it has no intrinsic resolution flaw, the resolution problems often arise only from the number of data that can reasonably be obtained. Thus, the basic total NMR signal recorded already represents some statistics over all the elementary signals; this property can be used to develop statistical approaches as described below.

Callaghan (1991) showed how the observation, by appropriate NMR measurements, of diffusion in simple liquids filling a porous system, may be used to characterize its structure (pore vs throat size, connectivity, etc). In the same spirit, it is possible to get a statistical information about the flow characteristics, thanks to the so-called pulse gradient field (PFG) NMR technique. The measurements provide the PDF (Probability Density Function), $f$, which is such as $f(v) d v$ is the probability to have a velocity between $v$ and $v+d v$. The trick behind this approach lies in that, for an appropriate variation of $\mathbf{G}$ in time (two opposite steps of duration $\delta$ and separated by a delay $\Delta>>\delta$ ), the phase shift in (2) writes as

$\phi=\gamma \int_{t} \mathbf{G}(t) \cdot \mathbf{r}(t) \mathrm{d} t \approx \nu w G \delta \Delta$

in which $v$ is the velocity component of the molecule along $\mathbf{G}$. Using $k=\gamma G \delta \Delta$ it follows that the total signal is proportional to

$S=\int m_{0} \exp (i k v) \mathrm{d} \mathbf{r}$

which may also be expressed as 
$S(k)=m \int_{v} f(v) \exp (i k v) d v$

We recognize the Fourier Transform of $f$, which can then be inverted to get $f$, if $S$ has been measured for a sufficient set of values of $k$. Moreover, the velocity distribution in any direction can be explored by an appropriate choice of $\mathbf{G}$. Let us recall that with this technique there is no problem of spatial resolution, and thus no impact of the pore size as long as the relaxation time is not too small, since in principle the signal related to the motion of any proton spin is recorded.

The PDF is an interesting marker of the flow in the porous medium, but it might be more appropriate to look at the distribution function, defined as $F(v)=\int_{-\infty}^{v} f(u) d u$, as the shape of $F$ is less dependent on the measurement uncertainties. For the flow of a Newtonian fluid through a simple cylindrical conduit, due to the parabolic velocity distribution and the amount of fluid proportional to the distance from the axis, $F$ is (theoretically) a straight line (see Figure 8). For a yield stress fluid flow with a central plug flow (see the inset of Figure 8a) this function is composed of two parts: a region of slow increase over the whole range of velocities and a peak at the maximum velocity value (corresponding to the plug) (see Fig.8a). By computing $F$ at different distances from the entrance of a yield stress fluid in a die we can thus see it changing from a straight line effectively associated with a parabolic velocity profile, to a yield stress fluid flow profile associated with a plug flow profile (see Fig.8a). This shows how this distribution can be interpreted in terms of velocity distribution in simple cases, but the function $F$ for the flow of a Newtonian fluid through a bead packing finally appears quite different: it is composed of a fast increase at low velocities followed by a long tail up to large velocities (see Figure 8 in which the corresponding PDF is also shown in the inset). Interestingly, such a distribution shape is approached by the theoretical one associated with the flow through a set of parallel conduits of different diameters (see Fig.8b), which shows that the classical approximation of a porous medium by a range of conduits (see e.g. Dullien (1992)) might be relevant at least for some flow characteristics.

This approach provides surprising results when used with complex fluids. Indeed, a similar function $F$, independent of the pore size, was found for a series of fluids exhibiting strongly different rheological behavior: a Newtonian, a shear-thinning, and a yield stress fluid (see Fig.8b). This in particular means that under these experimental conditions (flow induced by a piston), a negligible fraction of the yield stress fluid remains arrested (no step of $F$ is observed for $v=0$ ), in contrast with general expectations for a yield stress fluid flow through a system with a wide range of pore sizes. In order to explain the similarity of $F$ for the different types of fluids, it was suggested that in a system with rapidly varying boundary conditions (pore throat followed by expansion over a pore size length), the deformation is essentially imposed by the mean flow and the geometry of the pore, and not by the fluid properties (Bleyer and Coussot 2014). Such a phenomenon is finally similar to the observation of Figure 8a (inset) for the yield stress fluid entering the die: despite the strongly non-linear behavior of the fluid, it locally exhibits a Newtonian parabolic velocity profile. Although not often used so far it seems that this powerful technique could be used to get further original information on the flow characteristics of non-Newtonian fluids (Brown et al 2017) inside porous media. It was for example suggested that it could be used to predict some parameters of the pressure vs flow rate relationship (Chevalier et al 2014). PFG measurements coupled with imaging was also used within the frame of a study of a two-phase flow through a porous medium, providing a remarkable insight in the flow characteristics evolution during air intrusion (Rassi et al 2011). 

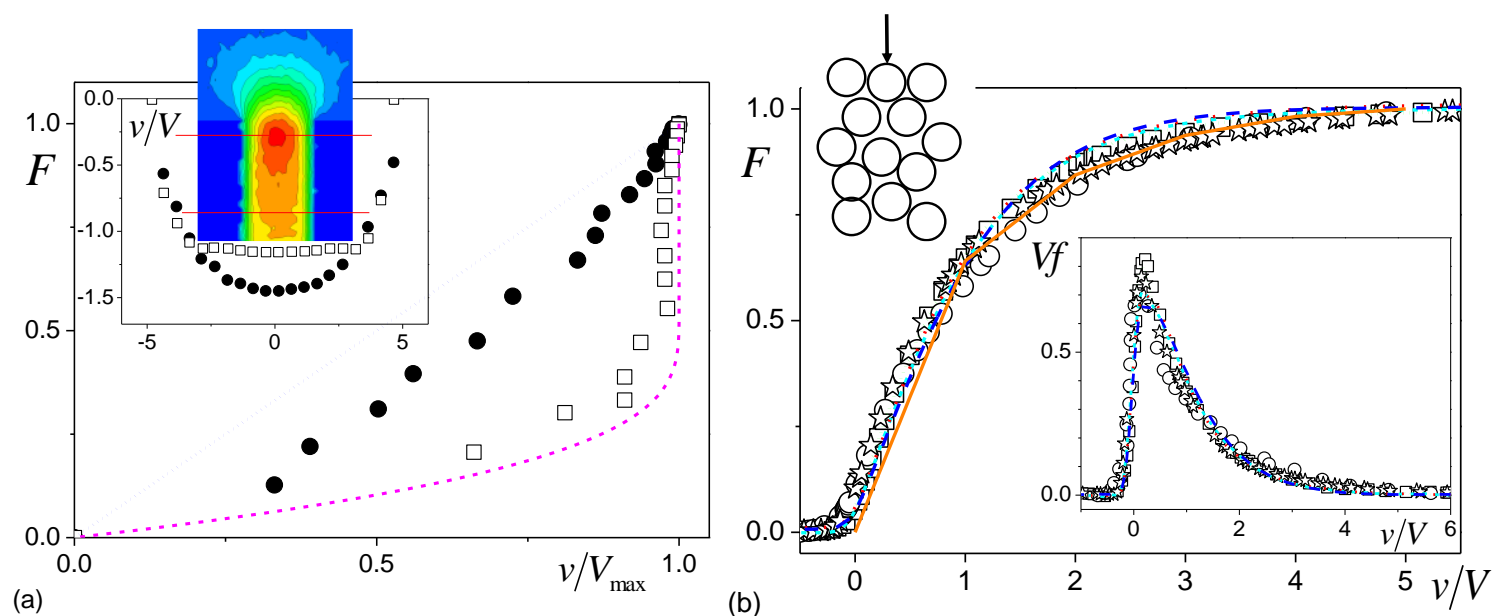

Figure 7: (a) Distribution function for the steady uniform flow of a Newtonian (blue dotted line) or yield stress fluid (magenta dashed line) through a conduit, and in two different cross-sections in a flow through a die imaged by MRI (data from Rabideau et al 2010): at the entrance in the die (circles) and at some distance from the entrance (squares); the inset shows the corresponding velocity profiles and the full velocity field. (b) Distribution function as a function of the velocity scaled by the average velocity through the voids for the flow through a bead packing as measured by MRI for: Newtonian fluids (squares (Lebon et al 1996) and circles Teyssier et al 1997), a powerlaw fluid (Xanthan) (Mertens et al 2006), and a yield stress fluid with different bead diameters and at different velocities (discontinuous lines) (see details in Chevalier et al 2014). The inset shows the corresponding PDF. The continuous line corresponds to a Newtonian fluid through 5 parallel conduits.

\subsection{Colloid transport}

\section{Transport and deposit}

Colloidal particles from industrial or natural sources propagate and may deposit and alter the environment they flow through. Common problems include the accumulation of particles impacting industrial (Cheremisinoff 1998), medical or biological processes (injection, filtration, storage, cleaning, sorting, etc.) or the leaking of contaminants from industrial or hydrologic processes transported in ground-water and accumulating in soils. Predicting particle transport and deposition by adsorption or clogging in these porous media is key to solve these problems. A large number of studies aimed at understanding the mechanisms of colloidal transport and adsorption, which may involve complex interactions between colloids, soil matrix, pore water and air (Masliyah and Bhattacharjee 2005). In most cases, the approaches rely on the interpretations of effluent concentration curves (particle "breakthrough curves") coming from columns of saturated porous media (Yao et al. 1971; Song and Elimelech 1993; Hahn et al. 2004; Simunek et al. 2006; Diaz et al. 2010). However such approaches provide only the final result of a complex phenomenon developing throughout the sample so that the full validation of a model is a difficult task. Thus, a significant step forward in the description of internal phenomena and validation of models can be expected from a detailed view of the mechanisms inside the porous medium. Since we are here dealing with transient two-phase flows one would ideally require to get information on the distribution of suspended and deposited particles at any time.

NMR has precisely the potential to provide the expected information. Indeed, although in the present context the NMR signal only originates from the spins of the protons of the liquid atoms, the local instantaneous concentration of particles can be determined from its impact on the relaxation times of the liquid phase. Indeed, the relaxation times decreases when the solid concentration 
increases as this implies more interactions between the liquid and the solid surfaces. In this context, during injection of suspension in a porous medium, Baumann and Werth (2005) obtained $T_{1}$ weighted images, from which they extracted the distribution of particle concentration in time, converting all MRI signal in concentration of suspended particles, despite an important adsorption. Ramanan et al. (2012), Lakshmanan et al. (2015a), and Lakshmanan et al. (2015b) obtained 2D $T_{2}$ weighted images of the liquid in coarse grain packings with an excellent resolution allowing to observe the transport in the structure at the pore scale, and deduced detailed characteristics of the evolution of suspended particle distribution in time along the sample. Alternatively, from a similar approach, the distribution of clogged particles for a suspension of non-colloidal particles flowing through a bead packing was measured by MRI at different times during the flow (Gerber et al 2018). Another original study focused on the impact of particle deposits on the flow characteristics and porous structure (seen through the PDF) (Fridjonsson et al 2014). Finally a further step was achieved by the work of Lehoux et al (2017 a), which provided both the adsorbed and suspended particle concentration distributions in time. This allowed to directly observe how the suspended particles progressively deposit along the medium in time, and provided a full set of data for testing the validity of models (Lehoux et al $2017 \mathrm{~b}$ ). This distinction of adsorbed and suspended particles could be achieved by taking into account that these two phenomena (concentration and adsorption) affect the relaxation time in different ways (Keita et al 2013). However the analysis of data through these techniques remains difficult and uncertain, so that it would still be very useful to develop more straightforward and precise approaches for measuring simultaneously the evolution of the deposited and flowing distributions of particles.

\section{Dispersion}

A specific problem of colloidal particle transport through porous media is mechanical dispersion, the process by which a solute or colloidal particles in a fluid progressively disperse wheres diffusion due to thermal agitation is negligible (Sahimi 2011, Kulasiri 2013). Several theoretical approaches have been developed (Bear 1988, Dullien 1992) mostly relying on the idea that since the structure is disordered each flowing element will follow a path similar to a random walk around the average velocity, a process leading on long times to Gaussian spreading. The basic parameter describing the process, namely the dispersion coefficient $(D)$, is often not precisely determined (Bear, 1988, Dullien 1992), in particular because, as for colloid transport, the standard description and quantification of the phenomenon basically rely on breakthrough curves. It was also suggested that imperfect flow injection could have a significant impact on the dispersion observed from breakthrough curve experiments (Scheven et al 2007). Actually, the dispersion coefficient can be directly measured from MRI visualizations of the transport characteristics of a pulse of paramagnetic nanoparticles. 2D imaging makes it possible to observe a homogeneous dispersion inside the sample, and to distinguish and leave apart entrance or exit effects which may induce significant radial heterogeneities (see Figure 8a). A more quantitative MRI approach can then provide quantitative measurements of the evolution in time of the longitudinal particle distribution in the sample (see Figure 8b). These data can be analyzed to deduce the intrinsic coefficient of dispersion, which appears to be smaller than the prediction from standard (macroscopic) tests (see Figure $8 \mathrm{~b}$ ).

In the other hand NMR PFG techniques can directly measure the statistics on molecular displacements in a flow over a given time interval, which provides a powerful technique to observe dispersion inside random bead packings. Generally, after travelling a distance of 10 to 20 bead diameters the displacement statistics in the main flow direction exhibits a Gaussian shape reminiscent of some asymptotic dispersion regime (Harding and Baumann 2001, Seymour and Callaghan 1997, Khrapitchev et al 2002, Stapf et al 1998, Codd and Seymour 2012). It is then possible to deduce the value of the dispersion coefficient, which also appears smaller than that generally deduced from macroscopic measurements by a factor between 2 and 10 (Seymour and Callaghan 1997, Lebon et al 1997, Deurer et al 2004, Khrapitchev and Callaghan 2003, Guillon et al 2013). 


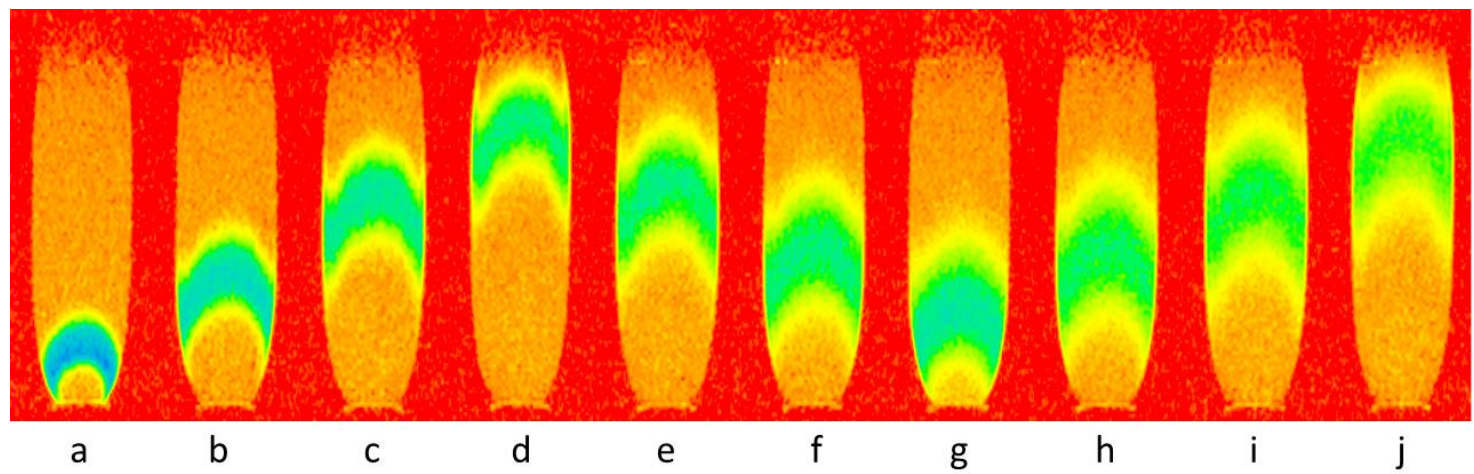

(A)

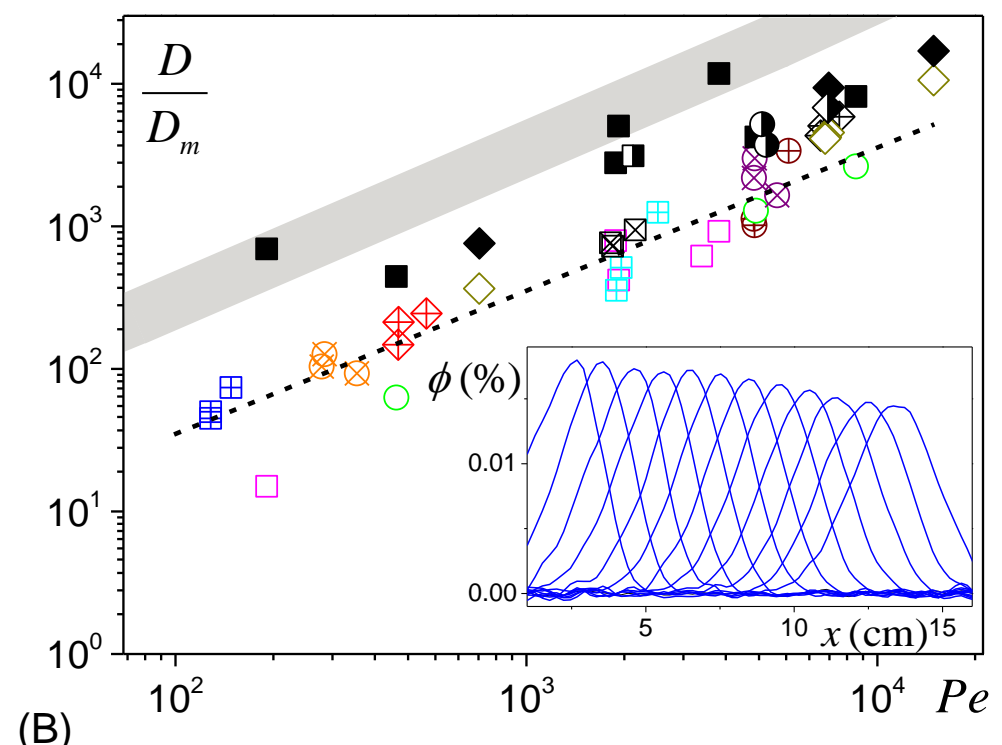

Figure 8: Transport and dispersion of a pulse of colloid particle suspension (surrounded by pure liquid) along a bead packing ( $570 \mu \mathrm{m}$ diameter). Flow direction is inverted several times before the pulse exi. (A) Images of the particle distribution along longitudinal cross-sections at successive times: first upstream flow $(a, b, c, d)$, downstream flow $(e, f, g)$, second upstream flow $(h, i, j)$. Color scale from maximum concentration to zero: blue-green-yellow-orange. (B) Dispersion coefficient scaled by the diffusion coefficient $\left(D_{m}\right)$ of the particles computed from the 1D NMR profiles (see inset) for different tests (open or crossed symbols) as a function of the Peclet number ( $P e=V d / D_{m}$ with $V$ the fluid velocity through pores and $d$ the grain diameter) with different porous materials: sand (squares), small beads (circles), large beads (diamonds). The grey area represents the region covered by data obtained by conventional techniques as gathered by Khrapitchev et al (2002), which covers the data leading to the "universal" flow curve as for example shown in Bear (1988) or Dullien (1992) and various other data obtained more recently. Dispersion coefficients determined from breakthrough curves for the same MRI tests are represented by filled symbols (single flow) and half-filled symbols (two flow reversals), directly illustrating the discrepancy. The inset shows 1D quantitative longitudinal profiles during the first upward flow shown in (a) as measured with another NMR technique. The dashed line is a guide for the eye drawn through the MRI data. Figure (A): Copyright Figure 2 of Lehoux et al 2016. Figure (B): Data from Lehoux et al (2016). 


\section{Transient liquid flows}

MR density imaging offers the possibility to observe the evolution of the liquid distribution in a porous medium as long as this distribution evolves sufficiently slowly. This makes it possible to get information on transient flows or phase changes (freezing or evaporation). Drying or imbibition are such processes, during which the liquid often slowly penetrates or is extracted from the porous medium, which can let sufficient time to get precise data of the "instantaneous" (as compared to the time scale of the process) liquid distribution in time. These successive distributions may be analyzed to deduce useful information on the transport characteristics. As for colloidal transport such information represents an important step forward to evaluate the validity of numerical models, an evaluation which otherwise relies on the analysis of the (global) drying or imbibition rate.

In that field, it is usual to measure directly the liquid distribution along the sample axis. This consists to determine the amount of liquid in thin cross-sectional layers of the sample along its axis. Under these conditions the spatial resolution of the obtained profiles is much better than for 2D images and one can directly analyze the data providing a straightforward quantitative description of the liquid transport in time. However, this approach is relevant only if the sample and the process are homogeneous in each cross-section. As a consequence, it can useful to follow the same time the process evolution from 2D imaging to check this homogeneity.

1D imaging of transient flows with MRI seems to start with the work by Pel et al (1996) who provided the water content distribution in time during drying of bricks and gypsum. Then MRI has been used in the same way for various materials: heated gypsum (Van der Heijden et al 2011), alkyd coatings (Erich et al 2006), fired-clay bricks (van der Heijden et al 2009), clay-grain-water pastes (Faure and Coussot 2010), plaster (Seck et al 2015, Seck et al 2016), nanocolloidal gels (Thiery et al 2015, 2016). These experiments in particular confirmed that for a sufficiently slow drying the liquid distribution remains uniform in a first regime lasting to a relatively low saturation (i.e. the liquid to pore volume ratio), then a dry front progressively penetrates the sample. Such an effect is due to capillary reequilibration processes, which induce liquid transport towards the region where the liquid has been extracted (free surface of the sample) and where the Laplace pressure is the lowest. By this means the free surface remains sufficiently wet for a long time, which explains that the drying rate is constant in the first regime. The saturation limit between the regime of uniform water distribution and the dry front regime depends on the material characteristics and the external conditions. Systematic measurements on uniform bead packings later showed that this scheme remains valid for pore size down to nanometer (Thiery et al 2017) although standard capillary effects are likely no more effective at this scale.

Actually MRI appears to be rather valuable to observe more complex drying effects in composite systems made of layers of different material types, as it allows to measure the transport of liquid through the different regions. In that aim we need to have layers perpendicular to the sample axis so that we can integrate the liquid distribution profile over the different regions to get the water content variation (and thus the drying rate) in each region (see Fig.9). This for example makes it possible to understand the mechanisms of drying of a paste covering a simple porous medium, as encountered with mortars or plasters coating building structures, or poultices used for removing salts from cultural heritage construction. We may thus observe (i) the extraction of the water (desaturation) from the porous medium situated below (and thus not directly exposed to ambient air) while the paste remains saturated (filled with water), (ii) the desaturation of the paste and finally (iii) the development of a dry front in both regions (see Figure 9). Remarkably, the drying rate remains constant over most of the duration of the process, i.e. whatever the region mainly drying, which means that the liquid is continuously transported towards the free surface of the sample to maintain a constant rate of evaporation. This is again a proof of the predominance of capillary effects during the process. The technique has also been used for plaster-brick (Nunes et al 2017, Petkovic et al 2007) or plaster-sandstone systems (Petkovic et al 2007), and even for a three-layer system (Petkovic et al 2010). A similar approach was used for describing the imbibition of liquid from a paste 
into an initially dry bead packing (Hazrati et al 2002, Fourmentin et al 2017, Ben Abdelouahab et al 2019).

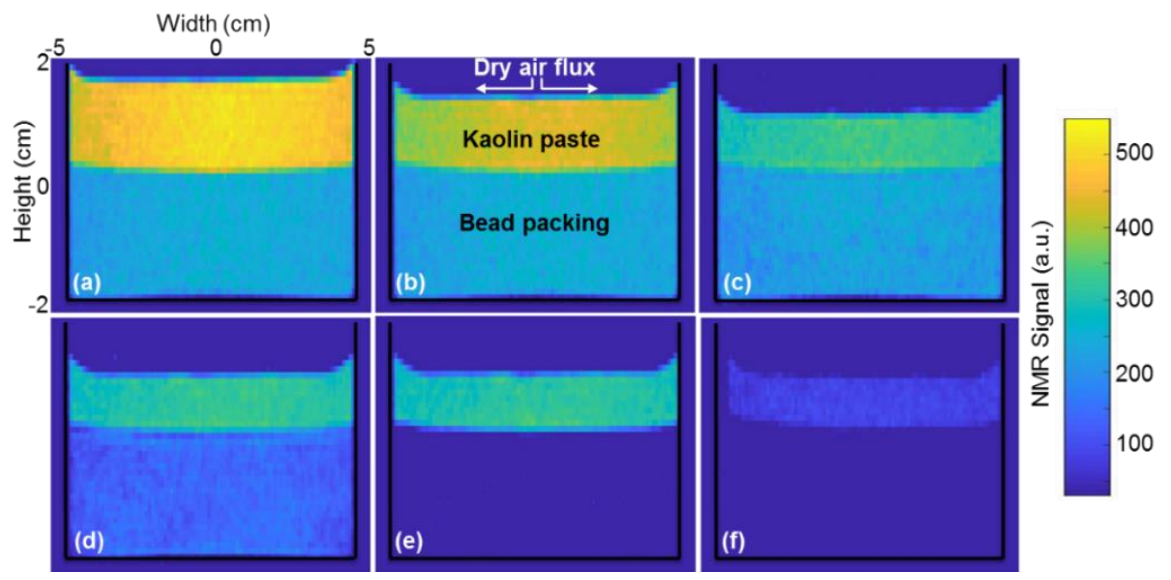

(A)

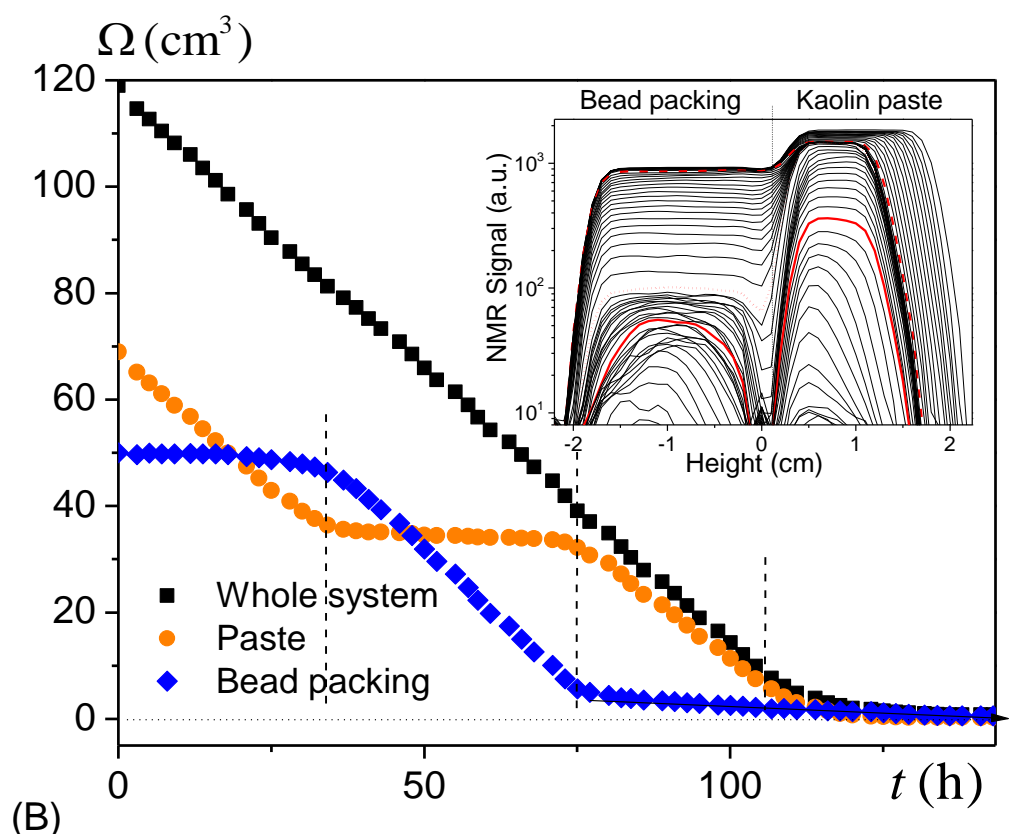

Figure 9: Convective drying of a kaolin paste (top) over a bead packing (bottom) initially saturated with water, with a dry air flux imposed along the sample top (height $=2.2 \mathrm{~cm}$ ). (A) Qualitative (T2-weighted) 2D MRI Images at different times: (a) 0, (b) $16 \mathrm{~h}$, (c) $34 \mathrm{~h}$, (d) $52 \mathrm{~h}$, (e) $75 \mathrm{~h}$, (f) $100 \mathrm{~h}$. The NMR signal on the color scale is for each phase an increasing, but non-linear, function of the local water amount. (B) Water volume evolution in the different parts of the system, estimated by integrating the NMR signal over the current height of each part of the successive NMR profiles. The inset shows the water amount profiles (from top to bottom) along sample axis at different times (time interval $2 \mathrm{~h}$ ). The dotted lines show the points of transition of regimes, and are associated with the red marked profiles in the inset. Copyright: Figures 4-5-6 of Ben Abdelouahab et al 2019 a

Since drying generally induces liquid transport thanks to capillary effects, the elements possibly suspended in the liquid can be transported and accumulated in some particular region of the medium, except if they sufficiently rapidly re-disperse by diffusion (Pel). This situation occurs for 
salts, which can lead to efflorescence (crystallization outside the sample) or subflorescence (crystallization inside). In this context, from Na-Imaging it appeared possible to follow the evolution of the crystallized salt in a drying brick (see Figure 10), limestone and other porous materials (Saidov et al 2015, 2017, Petkovic et al 2010). In parallel the authors checked that the liquid distribution remained uniform during all the period studied, which means that the liquid is continuously transported towards the free surface of the sample (see the concentration gradient at the approach of the sample free surface in Figure 10). It may be observed that the ions are initially transported towards the surface where they crystallize, and efflorescence occurs, then they start to redistribute throughout the medium (see Figure 10). This may be explained by the fact that the drying rate decreases in time, which leaves more time to ions to diffuse in the sample in the second regime. The distribution of ion concentration can also be determined through their impact on the NMR relaxation time, which for example made it possible to observe the electrodissolution of metal ions (Bray et al 2016). In a similar way, the liquid distribution and deposited particle distribution in time were also obtained in the case of drying of a colloidal suspension in a porous medium (Keita et al 2013, 2016). These data allowed to validate a simple model assuming in that case that each particle exactly follows the liquid path until the liquid evaporates (so that the particle adsorbs to the wall), or the particle is blocked by previously accumulated particles.

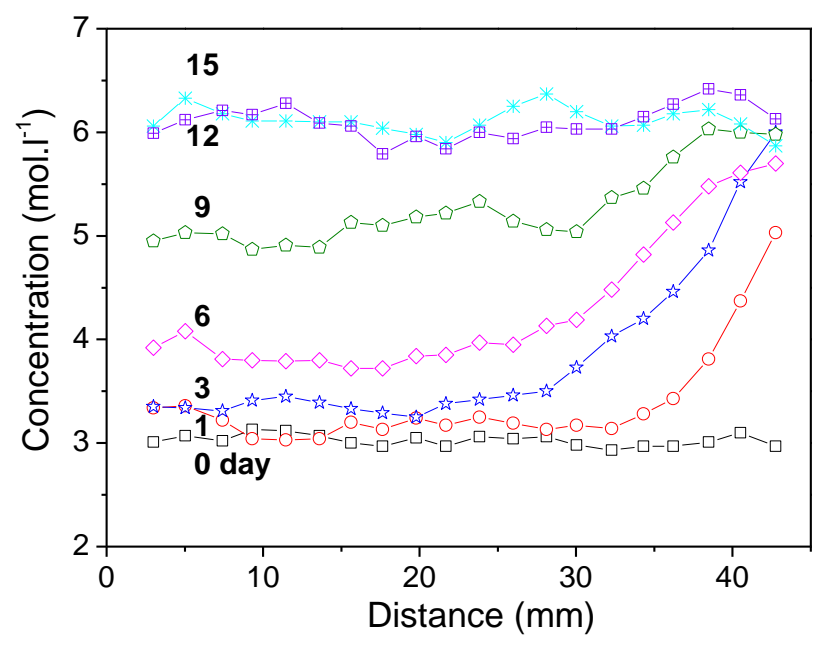

Figure 10: $\mathrm{NaCl}$ concentration profiles determined by MRI during drying of a fired-clay brick sample after different times. The sample free surface is on the right of the graph. Data from Pel et al (2002)

\section{Transfers in complex porous media}

Further outstanding measurement capabilities of NMR appear for the study of liquid transfers inside complex porous systems containing several types of pores and/or in which the liquid can be in different states. The simplest case is that of porous media containing pore classes of different sizes as may be encountered with building materials or soils. Apart from the above standard measurement of the (total) liquid distribution in time during slow processes such as drying, NMR allows to get information about the liquid distribution at a local scale in time, such as how the different pore classes are filled and how the liquid is distributed in each pore type. This is achieved from NMR relaxometry, or Time Domain NMR. This consists to determine the PDF of relaxation times, i.e. the density of probability to have a relaxation time in the different possible ranges. The principle of the technique is to look at the relaxation of the total NMR signal in the sample which, from (2), expresses as:

$S(t)=\int m_{0}(\mathbf{r}) \exp [-t / T] \mathrm{d} \mathbf{r}$ 
during its relaxation. In this expression the relaxation time may vary from one point to another in the sample, so that we can express the total signal in a different form:

$$
S(t)=m \int f(T) \exp [-t / T] \mathrm{d} T
$$

in which $m$ is the total NMR signal from the sample before any relaxation and $f$ the probability density function of the relaxation time $T$. The expression (7) can then be rearranged in the form of a Laplace transform of the function $f(u) / u^{2}$ (in which $u=1 / T$ ). Thus, from a series of measurements of $S$ at different times and then an inverse Laplace transform of $S$, we can finally get $f$ (see an example of such distributions in Figure 11).

Basically the technique provides a view of the different relaxation times of the liquid in a porous medium, and thus gives an idea of the fractions of the different pore sizes if they are saturated. Roughly speaking the relaxation time is related to the mobility of water molecules, and specific interactions of water with their environment (e.g. adsorption, proton exchange with other species, or magnetic interactions at nanoscale), so that, globally, the relaxation decreases when the liquid is more confined. In the particular case of water embedded in a pore cavity, within the usual hypothesis of biphasic fast exchange (Philippot et al 1998), the relaxation time scales as the ratio of the volume of free liquid water to the area of the water-solid interface, with a factor depending on the NMR surface relaxivity. Note that this volume to surface ratio is proportional to radius in the case of uniform spherical pores. Under these condition we expect that a pore is partially saturated, the relaxation time will also depend on the way the liquid is distributed in the pore (e.g. covering the solid surface or occupying a compact volume in a specific region of the pore).

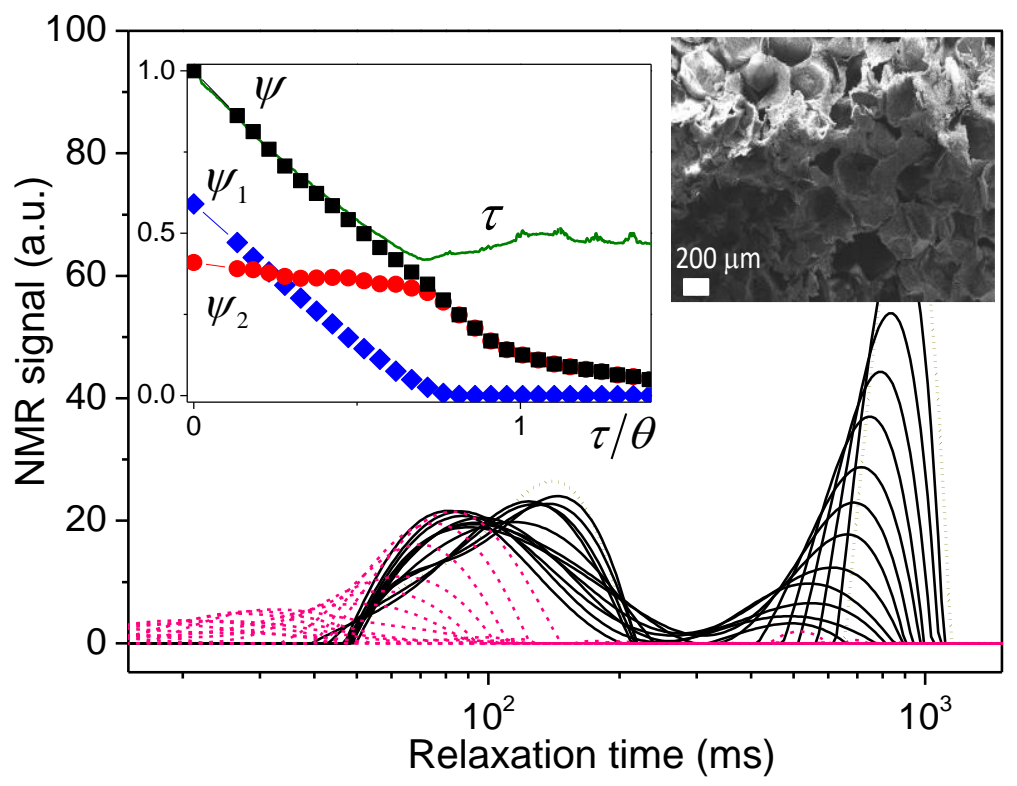

Figure 11: Convective drying of a bi-porous medium made of large inclusions dispersed in a small pore matrix (large pore to total pore volume ratio: 0.59) (see SEM (Scanning Electron Microscope) view of this medium in right inset): relaxation time distributions at different successive times (every $13 \mathrm{~min}$ ) (from right to left) during drying, first as black continuous lines, then as pink dashed lines from the last distribution reflecting liquid in large pores. The initial curve is drawn as a dotted line. The left inset shows the liquid fractions in the different regions (black squares for the total liquid fraction, blue diamonds for the liquid fraction in large pores, red circles in small pores) and the liquid phase contraction rate (green continuous line) as a function of the dimensionless time ( $\theta=17800 \mathrm{~s}$ ). Copyright Figure 8 of Lerouge et al 2020. 
The next step consists to look at the evolution of this relaxation time distribution in time. For example, from the distribution of relaxation times in a compressible biporous material during drying, it may be deduced that the large pores empty first, due to their compression, while the small pore matrix also slightly shrinks. This is evidenced by the fact that the peak associated with the large pores progressively decreases and shifts towards smaller relaxation times (see Figure 11), indicating that the amount of liquid in these large pores decreases, but their size decreases too. In the next step the small pores start to empty, but in that case no more contraction of the medium is observed while we again see a decrease of the corresponding peak in the distribution and a shift towards smaller relaxation times. Here, this results from the fact that the small pores drain homogeneously as for the drying of homogeneous solid porous media described in the previous section, thanks to capillary effects. The liquid then forms films along the pore walls, which progressively thin, inducing a relaxation time decrease. Note that during most of the time a constant drying rate is observed (see Figure 11), which is consistent with the above description since the homogeneous desaturation is associated with a liquid transport towards the sample free surface.

This technique can be used in even more complex systems, containing water in different phases. This is typically the case of bio-based composite construction materials, made of a cementitious matrix in which biomaterial pieces such as wood or hemp are dispersed. Under the condition that water (free or bound) in the matrix exhibits a relaxation time sufficiently different from bound or free water in the biomaterial inclusions (otherwise the two peaks overlap and form one single peak), it is possible to follow the transfers of liquid during the different stages of evolution of such systems: liquid extraction from bio-inclusions to favour cement setting (Faure et al 2012), drying, adsorption of water in bio-inclusions (Fourmentin et al 2016), liquid transport through the matrix, etc.
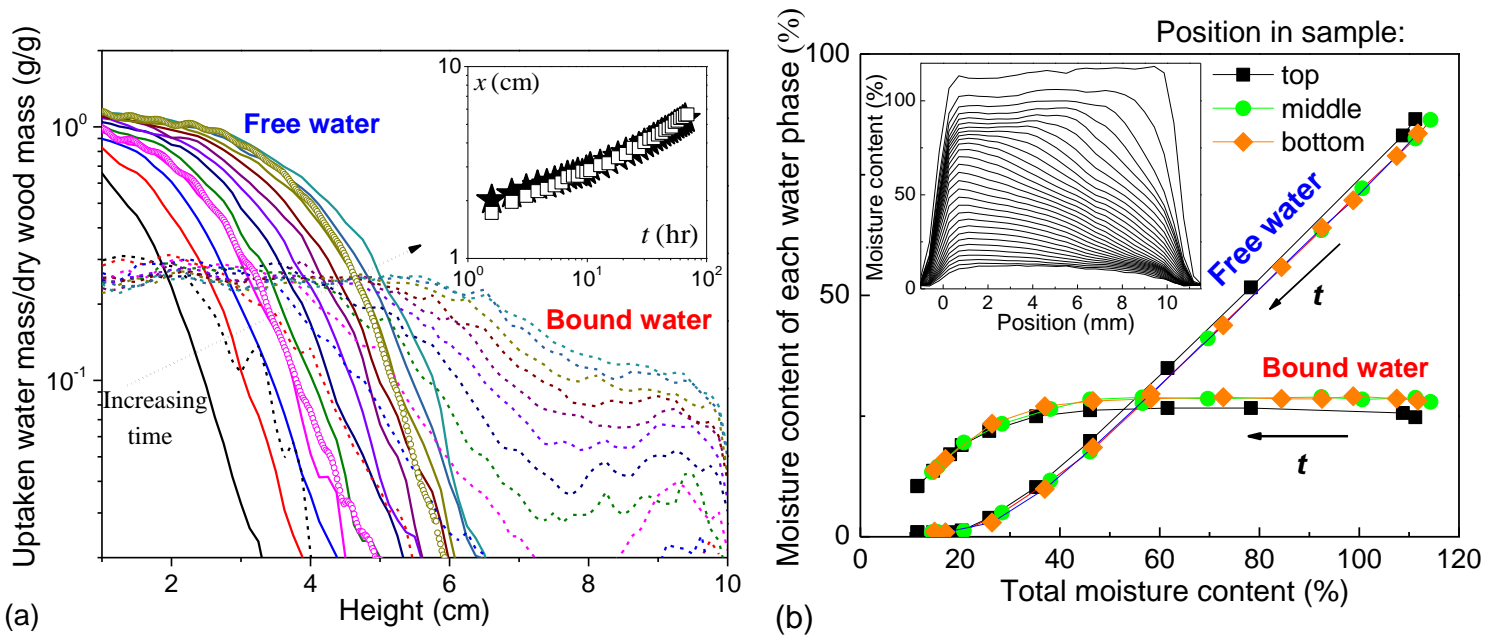

(b)

Figure 12: Water transfers in hardwood observed by NMR: (a) Imbibition in poplar; distribution of absorbed free water (solid lines) and bound water (short dash) content along the sample axis at different times; (from left to right) $3 \mathrm{~h}$ after first contact with water, then every $6 \mathrm{~h}$; the inset shows the distance reached from bottom by the mean position of the front of the free water profiles (taken at $0.1 \mathrm{~g} / \mathrm{g}$ ) (stars) and the end of the uniform bound water region (squares) (the uncertainty on this measurement is \pm $1.25 \mathrm{~mm}$ ); free water can thus apparently progress only when the wood is saturated with bound water. Copyright Figure 9 of Zhou et al 2018. (b) Drying of an oak sample; free and bound water content as a function of the (decreasing) moisture content in time; the inset shows the apparent moisture content distribution in time (top to bottom) (one profile every 6.8 h). Data from Gezici-Koç et al 2017. 
Imaging and relaxometry have been coupled to study the liquid transport in complex materials such as wood. Wood indeed contains different types of water with very different values of relaxation times: bound water, inside cell-walls, which exhibits relaxation times of the order of a few milliseconds, and free water in the tracheids (for softwood) or in the vessels and fibers (for hardwood), which typically exhibits a relaxation time of the order of one or several hundreds of milliseconds. Bound water can diffuse along cell-walls and free water can be transported along vessels or tracheids, or absorbed in cell-walls. This implies that the absorption (imbibition) or extraction (drying) of water from wood can involved these different phenomena through complex processes. In recent years it appeared possible to follow the evolution in time of the distributions of bound and free water individually along the sample (van Meel et al 2011, Dvinskikh et al 2011, Kekkonen et al 2013, Gezici-Koç et al 2017, Zhou et al 2018), which provided a critical insight in the understanding of the processes. It was thus found that during spontaneous imbibition, in contrast to porous media with an impermeable solid structure, the water imbibition in the hydraulic conduits of hardwood is not simply driven by the standard capillary effects associated with a good wetting of the solid surface, but it is in fact strongly affected by the adsorption of bound water in cell walls (Zhou et al 2019). More precisely bound water appears to progress far beyond the front of free water, and the free water penetration along the sample axis apparently coincides with the development of a region saturated with bound water (see Figure 12 a) (Zhou et al 2018). This likely explains that water imbibition is about three orders of magnitude slower than expected from standard Washburn imbibition process (Zhou et al 2019). The inverse effect seems to occur during drying (Gezici-Koç et al 2017): bound water is extracted only when all free water has been extracted (see Figure 12 b), even from pores (such as fibers) in which the liquid is not connected to the free surface through a hydraulic network, which suggests that bound water is the vector of this extraction.

\section{Conclusion}

We have seen that, thanks to its versatility, NMR already allowed a series of significant progress in fluid mechanics, in particular in the rheology of concentrated suspensions and for the study of transport or transfers in porous media. However, in several places it was suggested that the possibilities of NMR are far from having been fully exploited. This is for example the case for the statistical approach of fluid flows in porous media, for measurement of solid transport and deposit in porous media, for fluid transfers in complex materials, for the study of thixotropic fluid flows. It is likely that future works will allow the development of techniques for measuring simultaneously several physical parameters of the flows, thus allowing even more powerful characterization of the phenomenon.

\section{References}

Abbott JR, Tetlow N, Graham AL, Altobelli SA, Fukushima E, Mondy LA, Stephens TS (1991) Experimental observations of particle migration in concentrated suspensions: Couette flow, J. Rheol., 35:773-795

Al-kaby RN, Jayaratne JS, Brox TI, Codd SL, Seymour JD, Brown JR (2018) Rheo-NMR of transient and steady state shear banding under shear startup, J. Rheol., 62:1125-1134

Andrade DEV, Ferrari M, Coussot $P$ (2020) The liquid regime of waxy oils suspensions: A magnetic resonance velocimetry analysis, J. Non-Newt. Fluid Mech., 279:104261

Banko AJ, Benson MJ, Gunady IE, Elkins CJ, Eaton JK (2020) An improved three-dimensional concentration measurement technique using magnetic resonance imaging, Experiments in Fluids, 61:53 
Barnes E, Wilson D, Johns M (2006) Velocity profiling inside a ram extruder using magnetic resonance (MR) techniques, Chem. Eng. Sci., 61:1357-1367

Barrie PJ (2000) Characterization of porous media using NMR methods, Annual Reports on NMR spectrometry, 41:265-316

Baumann T, Werth CJ (2005) Visualization of colloid transport through heterogeneous porous media using magnetic resonance imaging, Colloids and Surfaces A, 265:2-10

Bear J (1988) Dynamics of fluids in porous media, Dover, New York

Ben Abdelouahab N, Gossard A, Rodts S, Coasne B, Coussot P (2019 a) Convective drying of a porous medium with a paste cover, Eur. Phys. J. E, 5:66

Ben Abdelouahab N, Gossard A, Rodts S, Coussot P (2019 b) Controlled imbibition from a soft wet material (poultice), Soft Matter, 15, 6732-6741 (2019)

Bleyer J, Coussot P (2014) Breakage of non-Newtonian character in flow through a porous medium: Evidence from numerical simulation, Phys. Rev. E, 89:063018

Bleyer J, Maillard M, de Buhan P, Coussot P (2015) Efficient numerical computations of yield stress fluid flows using second-order cone programming, Computer Methods in Applied Mechanics and Engineering, 283:599-614

Blythe TW, Sederman AJ, Mitchell J, Stitt EH, York APE, Gladden LF (2015) Characterizing the rheology of non-Newtonian fluids using PFG-NMR and cumulant analysis, J. Magnetic Resonance, 255:122-131

Bray JB, Davenport AJ, Ryder KS, Britton MM (2016) Quantitative, in situ visualization of metal-ion dissolution transport using ${ }^{1} \mathrm{H}$ Magnetic Resonance Imaging, Angewandte Chemie Int. Ed., 55:93949397

Britton MM, Callaghan PT (1997 a) Two-phase shear-band structures at uniform stress, Phys. Rev. Lett., 78:4930-4933

Britton MM, Callaghan PT (1997 b) NMR visualization of anomalous flow in cone-and-plate rheometry, J. Rheol., 41:1365-1386

Britton MM, Callaghan PT (1999) Shear-Banding instability in wormlike micellar solutions, European Physical J. B, 7:237-249

Brown JR, Fridjonsson EO, Seymour JD, Codd SL (2009) Nuclear magnetic resonance measurement of shear-induced particle migration in Brownian suspensions, Phys. Fluids, 21:093301

Brown JR, Trudnowski J, Nybo E, Kent KE, Lund T, Parsons A (2017) Quantification of non-Newtonian fluid dynamics of a wormlike micelle solution in porous media with magnetic resonance, Chem. Eng. Sci., 173:145-152

Callaghan PT (1991) Principles of nuclear magnetic resonance microscopy, Clarendon Press, Oxford Chaparian E, Frigaard IA (2017) Yield limit analysis of particle motion in a yield-stress fluid, J. Fluid Mech., 819:311-351

Cheremisinoff NP (1998) Liquid Filtration, Butterworth-Heinemann, Boston

Chernoburova O, Jenny M, Kiesgen De Richter S, Ferrari M, Otsuki A (2018) Dynamic behavior of dilute bentonite suspensions under different chemical conditions studied in Magnetic Resonance Imaging velocimetry, Colloids Interfaces, 2:41

Chevalier T, Rodts S, Chateau X, Boujlel J, Maillard M, Coussot P (2013) Boundary layer (shear-band) in frustrated viscoplastic flows, EPL, 102:48002

Chevalier T, Rodts S, Chateau X, Chevalier C, Coussot P (2014) Breaking of non-Newtonian character in flows through porous medium, Phys. Rev. E, 89:023002

Chow AW, Sinton SW, Iwamiya JH, Stephens TS (1994) Shear-induced particle migration in Couette and parallel-plate viscometers: NMR imaging and stress measurements, Phys. Fluids, 6, 2561-2576 
Codd SL, Seymour JD (2012) Nuclear magnetic resonance measurement of hydrodynamic dispersion in porous media: preasymptotic dynamics, structure and nonequilibrium statistical mechanics, Eur. Phys. J. Appl. Phys., 60:24204

Colbourne AA, Blythe TW, Barua R, Lovett S, Mitchell J, Sederman AJ, Gladden LF (2018) Validation of a low field Rheo-NMR instrument and application to shear-induced migration of suspended noncolloidal particles in Couette flow, J. Magn. Resonance, 286:30-35

Coleman BD, Markovitz H, Noll W (1966) Viscometric flows of non-Newtonian fluids, Springer Verlag, New York

Corbett AM, Phillips RJ, Kauten RJ, McCarthy KL (1995) Magnetic resonance imaging of concentration and velocity profiles of pure fluids and solid suspensions in rotating geometries, J. Rheol., 39:907924

Coussot P (2005) Rheometry of pastes, suspensions and granular materials, Wiley, New York

Coussot P (2014) Yield stress fluid flows: a review of experimental data, Journal of Non-Newtonian Fluid Mechanics, 211:31-49

Coussot P, Nguyen QD, Huynh HT, Bonn D (2002 b) Viscosity bifurcation in thixotropic, yielding fluids. Journal of Rheology, 46:573-589

Coussot P, Ovarlez G (2010) Physical origin of shear-banding of jammed systems, European Physical Journal E, 33:183-188

Coussot P, Raynaud JS, Bertrand F, Moucheront P, Guilbaud JP, Huynh HT, Jarny S, Lesueur D (2002 a) Coexistence of liquid and solid phases in flowing soft-glassy materials, Physical Review Letters, 88:218301

Coussot P, Tocquer L, Lanos C, Ovarlez G (2009) Macroscopic vs local rheology of yield stress fluids, Journal of Non-Newtonian Fluid Mechanics, 158:85-90

Deurer M, Vogeler I, Clothier BE, Scotter DR (2004) Magnetic resonance imaging of hydrodynamic dispersion in a saturated porous medium, Transport in Porous Media, 54, 145-166

Diaz J, Rendueles M, Diaz M (2010) Straining phenomena in bacteria transport through natural porous media. Environ. Sci. Pollut. Research, 17:400-409

Dimitriou CJ, McKinley GH (2019) A canonical framework for modeling elasto-viscoplasticity in complex fluids, J. Non-Newt. Fluid Mech., 265:116-132

Douglass BS, Colby RH, Madsen LA, Callaghan PT (2008) Rheo-NMR of wormlike micelles forms from nonionic Pluronic surfactants, 41:804-814

Dullien FAL (1992) Porous media - Fluid transport and porous structure, Academic Press, San Diego

Durst F, Melling A, Whitelaw JH (1976) Principles and practice of laser-Doppler anemometry, Academic Press, New York

Dvinskikh SV, Henriksson M, Mendicino AL, Fortino S, Toratti T (2011) NMR imaging study and multiFickian numerical simulation of moisture transfer in Norway spruce samples, Engineering Structures, 33:3079-3086

Elkins CJ, Alley MT (2007) Magnetic resonance velocimetry: applications of magnetic resonance imaging in the measurements of fluid motion, Exp. Fluids, 43:823-858

Erich SJF, Laven J, Pel L, Huinink HP, Kopinga K (2006) NMR depth profiling of drying alkyd coatings with different catalysts, Progress in Organic Coatings, 55:105-111

Ernst RR, Bodenhausen G, Wokaun A (1987) Principles of Nuclear Magnetic Resonance in one and two dimensions, Oxford Science Publications, Oxford

Fardin MA, Lerouge S (2012) Instabilities in wormlike micelle systems: from shear-banding to elastic turbulence, Eur. Phys. J. E, 35:91

Faure $\mathrm{P}$, Coussot, $\mathrm{P}$ (2010) Drying of a model soil, Physical Review E, 82:036303 
Faure P, Peter U, Lesueur D, Coussot P (2012) Water transfers within Hemp Lime Concrete followed by NMR, Cement and Concrete Research, 42:1468-1474

Fourmentin M, Faure P, Pelupessy P, Sarou-Kanian V, Peter U, Lesueur D, Rodts S, Daviller D, Coussot $P$ (2016) NMR and MRI observation of water absorption/uptake in hemp shives used for hemp concrete, Construction and Building Materials, 124, 405-413

Fourmentin M, Faure P, Rodts S, Peter U, Lesueur D, Daviller D, Coussot P (2017) NMR observation of water transfer between a cement paste and a porous medium, Cement and Concrete Research, 95:56-64

Fraggedakis D, Dimakopoulos Y, Tsamopoulos J (2016) Yielding the yield stress analysis: a thorough comparison of recently proposed elastoc-visco-plastic (EVP) fluid models, J. Non-Newt. Fluid Mech., 238:170-188

Fridjonsson EO, Codd SL, Seymour JD (2012) Application of PFG-NMR to study the impact of colloidal deposition on hydrodynamic dispersion in a porous medium, Transp. Porous Med., 103:117-130

Fuller GG (1995) Optical rheometry of complex fluids, Oxford University Press, Oxford

Gallot T, Perge C, Grenard V, Fardin MA, Taberlet N, Manneville S (2013), Ultrafast ultrasonic imaging coupled to rheometry: principle and illustration, Rev. Sci. Instr. 84:045107

Geizici-Koç Ö, Erich JF, Huinink H, ven der Ven LGJ, Adan OCG (2017) Bound and free water distribution in wood during water uptake and drying as measured by $1 \mathrm{D}$ magnetic resonance imaging, Cellulose, 24:535-553

Gerber G, Rodts S, Aimedieu P, Faure P, Coussot P (2018) Particle-size exclusion regimes in porous media, Physical Review Letters, 120:148001

Gladden LF, Mitchell J (2011) Measuring adsorption, diffusion and flow in chemical engineering: applications of magnetic resonance to porous media, New J. Physics, 13:035001

Gladden LF, Sederman AJ (2013) Recent advances in flow MRI, J. Magn. Resonance, 229:2-11

Götz J, Kreibich W, Peciar M, Buggisch H (2001) MRI of Couette experiments in a newly developed shear device - suitable for pastes and concentrated suspensions, J. Non-Newt. Fluid Mech., 98:117139

Götz J, Zick K, Heinen C, König T (2002) Visualisation of flow processes in packed beds with NMR imaging: determination of the local porosity, velocity vector and local dispersion coefficients, Chem. Eng. Processing, 41: 611-629

Graham AL, Altobelli SA, Fukurshima E, Mondy LA, Stephens TS (1991) NMR imaging of shear-induced diffusion structure in concentrated suspensions undergoing Couette flow, J. Rheol., 35:191-201

Guillon V, Fleury M, Bauer D, Neel MC (2013) Superdispersion in homogeneous unsaturated porous media using NMR propagators, Phys. Rev. E, 87:043007

Haavisto, Cardona MJ, Salmela J, Powell RL, McCarthy MJ, Kataja M, Koponen Al (2017) Experimental investigation of the flow dynamics and rheology of complex fluids in pipe flow by hybrid multi-scale velocimetry, Experiments in Fluids, 58:158

Hahn MW, Abadzic D, O'Melia CR 52004) Aquasols: on the role of secondary minima, Environ. Sci. Technol., 38:5915-5924

Harding SG, Baumann H (2001) Nuclear Magnetic Resonance Studies of Solvent Flow Through Chromatographic Columns: Effect of Packing Density on Flow Patterns, J. Chromatography A, 905:1934

Hazrati K, Pel L, Marchand J, Kopinga K, Pigeon M (2002) Determination of isothermal unsaturated capillary flow in high performance cement mortars by NMR imaging, Materials and Structures 35:614-622

Hollingsworth KG, Johns ML (2004) Rheo-nuclear magnetic resonance of emulsion systems, J. Rheol., 48:787-803 
Holmes WM, Callaghan PT, Vlassopoulos D, Roovers J (2004) Shear-banding phenomena in ultrasoft colloidal glasses, J. Rheol., 48:1085-1101

Hunt AG, Skinner TE, Ewing RP, Ghandarian-Alavijeh B (2011) Eur. Phys. J. B, 80, 411

Iwamiya JH, Chow AW, Sinton SW (1994), NMR flow imaging of Newtonian liquids and a concentrated suspension through an axisymmetric sudden contraction, Rheol Acta, 33:267-282

Jarny S, Roussel N, Rodts S, Le Roy R, Coussot P (2005) Rheological behavior of cement pastes from MRI velocimetry, Concrete Cement Research, 35:1873-1881

Jossic L, Briguet A, Magnin A (2002) Segregation under flow of objects suspended in a yield stress fluid and NMR visualization, Chem. Eng. Sci., 57:409-418

Jossic L, Magnin A (2005) Structuring of gelled suspensions flowing through a sudden three-

dimensional expansion, J. Non-Newt. Fluid Mech., 127:201-212

Keita E, Faure P, Rodts S, Coussot P (2013) MRI evidence for a receding-front effect in drying porous media, Phys. Rev. E, 87:062303

Keita $E$, Kodger TE, Faure $P$, Rodts $S$, Weitz DA, Coussot $P$ (2016) Water retention against drying with soft particle suspensions in porous media, Phys. Rev. E , 94:033104

Kekkonen PM, Ylisassi A, Telkki VV (2013) Absorption of water in thermally modified pine wood as studied by Nuclear Magnetic Resonance, J. Phys. Chem., 118:2146-2153

Khrapitchev AA, Callaghan PT (2003) Reversible and irreversible dispersion in a porous medium Phys Fluids, 15, 2649-2660

Khrapitchev AA, Stapf S, Callaghan PT (2002) NMR visualization of displacement correlations for flow in porous media, Phys. Rev. E, 66, 051203

Kind J, Thiele CM (2019) MRI and localized NMR spectroscopy of sessile droplets on hydrophilic, hydrophobic and superhydrophobic surfaces - Examination of the chemical composition during evaporation, J. Magnetic Resonance, 307:106579

Koptyug IV, Sagdeev RZ (2002) Applications of NMR tomography to mass transfer studies, Russian Chemical Reviews, $71:$ 789-835

Krieger IM, Dougherty TJ (1959) A Mechanism for Non-Newtonian Flow in Suspensions of Rigid Spheres, Journal of Rheology, 3:137-145

Kulasiri D (2013) Non-Fickian solute transport in porous media, Springer, Berlin

Lakshmanan S, Holmes WM, Sloan WT, Phoenix VR (2015 a) Nanoparticle transport in saturated porous medium using magnetic resonance imaging, Chem. Eng. J., 266:156-162

Lakshmanan S, Holmes WM, Sloan WT, Phoenix VR (2015 b) Characterization of nanoparticle transport through quartz and dolomite gravels by magnetic resonance imaging, Int. J. Environ. Sci. Technol., 12:3373-3384

Lebon L, Leblond J, Hulin JP (1997) Experimental measurement of dispersion processes at short times using a pulsed field gradient NMR technique, Phys. Fluids, 9:481-490

Lebon L, Oger L, Leblond J, Hulin JP, Martys NS, Schwartz LM (1996) Pulsed gradient NMR measurements and numerical simulation of flow velocity distribution in sphere packings, Phys. Fluids, 8:293-301

Lehoux AP, Faure P, Lafolie F, Rodts S, Courtier-Murias D, Coussot P, Michel E (2017 b) Combined time-lapse magnetic resonance imaging and modeling to investigate colloid deposition transport in porous media, Water Research, 123:12-20

Lehoux AP, Faure P, Michel E, Courtier-Murias D, Rodts S, Coussot P (2017 a) Transport and adsorption of nano-colloids in porous media observed by Magnetic Resonance Imaging, Transport in Porous Media, 19:403-423

Lehoux AP, Rodts S, Faure P, Michel E, Courtier-Murias D, Coussot P (2016) MRI measurements evidence weak dispersion in homogeneous porous media, Phys. Rev. E , 94, 053107 
Lerouge T, Maillet B, Courtier-Murias D, Grande D, Le Droumaguet B, Pitois O, Coussot P (2020) Drying of a compressible biporous material, Phys. Rev. Applied, 13:044061

Lopez-Gonzalez MR, Holmes WM, Callaghan PT (2006) Rheo-NMR phenomena of wormlike micelles, Soft Matter, 2:855-869

Lovreglio P, Das S, Buist KA, Peters EAJF, Pel L, Kuipers JAM (2018) Experimental and numerical investigation of structure of hydrodynamics in packed beds of spherical particles, AIChE J., 64:18961907

Masliyah JH, Bhattacharjee S (2005) Electrokinetic and Colloid Transport Phenomena, Wiley, New York

Medronho B, Brown J, Miguel MG, Schmidt C, Olsson U, Galvosas P (2011) Planar lamellae and onions: a spatially resolved rheo-NMR approach to the shear-induced structural transformations in a surfactant model system, Soft Matter, 7:4938-4947

Mendes (de Souza) PR (2011) Thixotropic elasto-viscoplastic model for structured fluids, Soft Matter, 7:2471-2483

Mendes (de Souza) PR, Thompson RL (2012) A critical overview of elasto-viscoplastic thixotropic modeling, J. Non-Newt. Fluid Mech., 187:8-15

Mendes R, Vinay G, Ovarlez G, Coussot P (2015) Reversible and Irreversible Destructuring Flow in Waxy Oils: an MRI Study, J. Non-Newt. Fluid Mech., 220:77-86

Mertens D, Heinen C, Hardy EH, Buggisch HW (2006) Newtonian and non-Newtonian low Re number flow through bead packings, Chem. Eng. Technol., 29, 854-861

Milani PM, Gunady IR, Ching DS, Banko AJ, Elkins CJ, Eaton JK (2019) Enriching MRI mean flow data of inclined jets in crossflow with Large Eddy Simulations, Int. J. Heat Fluid Flow, 80:108472

Moller PCF, Rodts S, Michels MAJ, Bonn D (2008) Shear-banding an dyield stress in soft glassy materials, Phys. Rev. E, 77:041507

Moraczewski T, Tang H, Shapley NC (2005) Flow of a concentrated suspension through an abrupt axisymmetric expansion measured by nuclear magnetic resonance imaging, J. Rheol, 49:1409-1428

Nikolaeva T, Vergeldt FJ, Serial R, Dijksman JA, Venema P, Voda A, van Duynhoven J, Van As H (2020) High field micro MRI velocimetry measurement of quantitative local flow curves, Anal. Chem., 92:4193-4200

Nunes C, Pel L, Kunecky J, Slizkova Z (2017) The influence of the pore structure on the moisture ransport in lime plaster-brick systems as studied by NMR, Construction and Building Materials, 142:395-409

Ovarlez G, Bertrand F, Rodts S (2006) Local determination of the constitutive law of a dense suspension of non-colloidal particles through magnetic resonance imaging, J. Rheol., 50:259-292

Ovarlez G, Mahaut F, Bertrand F, Chateau X (2011) Flows and heterogeneities with a vane tool: Magnetic resonance imaging measurements, J. Rheol., 55:197-223

Ovarlez G, Rodts S, Chateau X, Coussot P (2009) Phenomenology and physical origin of shearlocalization and shear-banding in complex fluids, Rheologica Acta, 48:831-844

Ovarlez G, Rodts S, Ragouilliaux A, Coussot P, Goyon J, Colin A (2008) Wide-gap Couette flows of dense emulsions: Local concentration measurements, and comparison between macroscopic and local constitutive law measurements through magnetic resonance imaging, Physical Review $E$ 78:036307

Pavlovskaya GE, Meersmann T, Jin C, Rigby SP (2018) Fluid flow in a porous medium with transverse permeability discontinuity, Phys. Rev. Fluids, 3:044102

Pel L, Brocken H, Kopinga K (1996) Determination of moisture diffusivity in porous media using moisture concentration profiles, Int. J. Heat Mass Transfer, 39:1273-1280 
Pel L, Huinink H, Kopinga K (2002) Ion transport and crystallization in inorganic building materials as studied by nuclear magnetic resonance, Applied Physics Letters, 81:15.

Pel L, Huinink H, Kopinga K (2003) Salt transport and crystallization in porous building materials, Magn. Res. Imaging, 21:317-320

Petkovic J, Huinink HP, Pel L, Kopinga K, van Hees RPJ (2007) Salt transport in plaster/substrate layers, Materials and Structures, 40:475-490

Petkovic J, Huinink HP, Pel L, Kopinga K, van Hees RPJ (2010) Moisture and salt transport in threelayer plaster/substrate systems, Construction and Building Materials,24:118-127

Philippot S, Korb JP, Petit D, Zanni H (1998) Analysis of microporosity and setting of reactive powder concrete by proton nuclear relaxation, Magn. Reson. Imaging, 16:515-519

Rabideau BD, Moucheront P, Bertrand F, Rodts S, Melinge Y, Lanos C, Coussot P (2012) Internal flow characteristics of a plastic kaolin suspension during extrusion, J. Am. Ceram. Soc., 95:494-501

Rabideau BD, Moucheront P, Bertrand F, Rodts S, Roussel N, Lanos C, Coussot P (2010) The extrusion of a model yield stress fluid imaged by MRI velocimetry, J. Non-Newt. Fluid Mech., 165:394-408

Raffel M, Willert C, Kompenhans J (1998) Particle Image Velocimetry-A practical guide, Springer Verlag, Berlin

Ragouilliaux A, Herzhaft B, Bertrand F, Coussot P (2006) Flow instability and shear localization in a drilling mud, Rheol. Acta, 46:261-271

Ragouilliaux A, Ovarlez G, Shahidzadeh-Bonn N, Herzhaft B, Palermo T, Coussot P (2007) Transition from a simple yield stress fluid to a thixotropic material, Physical Review E, 76, 051408

Ramanan B, Holmes WM, Sloan WT, Phoenix VR (2012) Investigation of nanoparticle transport inside coarse-grained geological media using magnetic resonance imaging, Environ. Sci. Technol., 46:360366

Rassi EM, Codd SL, Seymour JD (2014) Corrigendum: Nuclear magnetic resonance characterization of the stationary dynamics partially saturated media during steady-state infiltration flow (2011 New J.

Phys. 13:015007), New J. Phys., 16:039501

Raynaud JS, Moucheront P, Baudez JC, Bertrand F, Guilbaud JP, Coussot P (2002) Direct determination by NMR of the thixotropic and yielding behavior of suspensions, Journal of Rheology, 46:709-732

Ren X, Stapf S, Blümich B (2005) NMR velocimetry of flow in model fixed-bed reactors of low aspect ratio, AIChE J., 51:392-405

Rodts S, Boujlel J, Rabideau BD, Ovarlez G, Roussel N, Moucheront P, Lanos C, Bertrand F, Coussot P (2010) Solid-liquid transition and rejuvenation similarities in complex flows of thixotropic materials studied by NMR and MRI, Physical Review E, 81:021402

Rogers SA, Callaghan PT (2009) Time-dependent NMR-velocimetry of a colloidal glass, Rheol. Acta, 48:735-745

Sahimi M (2011) Flow and Transport in Porous Media and Fractured Rock, Wiley-VCH Verlag GmbH \& Co. KGaA, Weinheim

Saidov TA, Pel L, Kopinga K (2017) Sodium sulfate salt weathering of porous building materials studied by NMR, Materials and Structures, 50:145

Saidov TA, Pel L, van der Heijden GHA (2015) Crystallization of sodium sulfate in porous media by drying at a constant temperature, Int. J. Heat Mass Transfer, 83:621-628

Salmon JB, Manneville S, Colin A, Pouligny B (2003) An optical fiber based interferometer to measure velocity profiles in sheared complex fluids Eur. Phys. J. AP, 22:143-154

Sankey MH, Holland DJ, Sederman AJ, Gladden LF (2009) Magnetic resonance velocity imaging of liquid and gas two-phase flow in packed beds, J. Magn. Resonance, 196:142-148 
Saramito P (2007) A new constitutive equation for elastoviscoplastic fluid flows, J. Non-Newt Fluid Mech., 145:1-14

Saramito P, Wachs A (2017) Progress in numerical simulation of yield stress fluid flows, Rheol Acta, $56: 211-230$

Scheven UM, Harris R, Johns ML (2007) Intrinsic dispersivity of randomly packed monodisperse spheres, Phys. Rev. Lett. 99:054502

Seck MD, Keita E, Faure P, Cavalié P, Van Landeghem M, Rodts S, Coussot P (2016) Subflorescence and plaster drying dynamics, Chem. Eng. Sci., 148:203-211

Seck MD, Van Landeghem M, Faure P, Rodts S, Cavalié P, Combes R, Keita E, Coussot P (2015) The mechanisms of plaster drying, J. Mat. Sci., 50:2491-2501

Sederman AJ, Johns ML, Alexander P, Gladden LF (1998) Structure-flow correlations in packed beds, Chem. Eng. Sci., 53:2117-2128

Sederman AJ, Mantle MD, Buckley C, Gladden LF (2004) MRI technique for measurement of velocity vectors, acceleration, and autocorrelation functions in turbulent flow, J. Magn. Resonance, 166:182189

Seymour JD, Callaghan PT (1997) Generalized approach to NMR analysis of flow and dispersion in porous media, AIChE J., 43, 2096-2111

Seymour JD, Callaghan PT (1997) Generalized approach to NMR analysis of flow and dispersion in porous media, AIChE J., 43, 2096-2111

Seymour JD, Maneval JE, McCarthy KL, Powell RL, McCarthy MJ (1995) Rheological characterization of fluids NMR velocity spectrum measurements, J. Texture Studies, 26:89-101

Seymour JD, Manz B, Callaghan PT (1999) Pulsed gradient spin echo nuclear magnetic resonance measurements of hydrodynamic instabilities with coherent structure: Taylor vortices, Physics of Fluids, 11:5

Shim G, Prasad D, Elkins CJ, Eaton JK, Benson MJ (2019) 3D MRI measurements of the effects of wind direction on flow characteristics and contaminant dispersion in a model urban canopy,

Environmental Fluid Mechanics, 19:851-878

Simunek, J., He, C., Pang, L., Bradford, S. A.: Colloid-facilitated solute transport in variably saturated porous media: numerical model and experimental verification, Vadose Zone J., 5 1035-1047 (2006)

Sinton SW, Chow AW (1991) NMR flow imaging of fluids and solid suspensions in Poiseuille flow, J. Rheol., 35:735-772

Skuntz ME, Perera D, Maneval JE, Seymour, Anderson R (2018) Melt-front propagation and velocity profiles in packed beds of phase-change materials measured by magnetic resonance imaging, Chem. Eng. Sci., 190:164-172

Song L, Elimelech M (1993) Dynamics of colloid deposition in porous media: modeling the role of retained particles, Colloid. Surface, A 73:49-63

Stapf S, Packer KJ, Graham RG, Thovert JF, Adler PM (1998) Spatial correlations and dispersion for fluid transport through packed glass beads studied by pulsed field-gradient NMR, Physical Review $E$, 58:6206

Teyssier JJ, Packer KJ, Thovert JF, Adler PM (1997) NMR measurements an dnumerical simulation of fluid transport in porous solids, AIChE J., 43, 1653-1661

Thiery J, Rodts S, Keita E, Chateau X, Faure P, Courtier-Murias D, Kodger TE, Coussot P (2015) Water transfer and crack regimes in nanocolloidal gels, Phys. Rev. E, 91:042407

Thiery J, Keita E, Rodts S, Courtier Murias D, Kodger TE, Pegoraro A, Coussot P (2016) Drying kinetics of deformable and cracking nano-porous gels, Eur. Phys. J. E, 39:117

Thiery J, Rodts S, Weitz DA, Coussot P (2017) Drying regimes in homogeneous porous media from macro- to nanoscale, Phys. Rev. Fluids, 2:074201 
Vallatos A, Wilson MCT, Taylor AF, Britton MM (2012) Characterizing stationary and translating vortex flow using magnetic resonance, EPL, 99:68001

van der Heijden GHA, Huinink HP, Pel L, Kopinga K (2009) Non-isothermal drying of fired-clay brick, an NMR study, Chem. Eng. Sci., 64:3010-3018

van der Heijden GHA, Pel L, Huinink HP, Kopinga K (2011) Moisture transport and dehydration in heated gypsum, an NMR study, Chem. Eng. Sci., 66:4241-4250

Van Meel PA, Erich SJF, Huinink H, Kopinga K, de Jong J, Adan OCG (2011) Moisture transport in coated wood, Progress in Organic Coatings, 72:686-694

Varchanis S, Haward SJ, Hopkins CC, Syrakos A, Shen AQ, Dimakopoulos Y, Tsamopoulos J (2020)

Transition between solid and liquid state of yield-stress fluids under purely extensional deformations, PNAS, 117:12611-12617

Varchanis S, Makrigiorgos G, Moschopoulos P, Dimakopoulos Y, Tsamopoulos J (2019) Modeling the rheology of thixotropic elasto-visco-plastic materials, J. Rheol., 4:609-639

Wassenius H, Callaghan PT (2005) NMR velocimetry studies of the steady-shear rheology of a concentrated hard-sphere colloidal system, Eur. Phys. J. E, 18:69-84

Wiederseiner S, Andreini N, Epely-Chauvin G, Moser G, Monnereau M, Gray J, Ancey C (2011) Experimental investigation into segregating granular flows down chutes, Physics of Fluids, $23: 013301$

Wiese M, Benders S, Blümich B, Wessling M (2018) 3D MRI velocimetry of non-transparent 3Dprinted staggered herringbone mixers, Chem. Eng. J., 343:54-60

Wood BD, Apte SV, Liburdy JA, Ziazi RM, He X, Finn JR, Patil VA (2015) A comparison of measured and modeled velocity fields for a laminar flow in a porous medium, Advances in Water resources, $85: 45-63$

Yao KM, Habibian MT, Omelia CR (1971) Water and waste water filtration. Concepts and applications. Environ. Sci. Technol., 5:1105-1112

Zhang X, Fadoul O, Lorenceau E, Coussot P (2018 a) Yielding and flow of soft-jammed systems in elongation, Physical Review Letters, 120:048001

Zhang $X$, Lorenceau E, Bourouina T, Basset $P$, Oerther T, Ferrari M, Rouyer F, Goyon J, Coussot $P$ (2018 b) Wall slip mechanisms in direct and inverse emulsions, J. Rheol. 62:1495-1513

Zhou M, Caré S, Courtier-Murias D, Faure P, Rodts S, Coussot P (2018) Magnetic resonance imaging evidences of the impact of water sorption on hardwood capillary imbibition dynamics, Wood Science and Technology, 52:929-955

Zhou M, Caré S, King A, Courtier-Murias D, Rodts S, Gerber G, Aimediue P, Bonnet M, Bornert M, Coussot $P$ (2019) Wetting enhanced by water adsorption in hygroscopic plantlike materials, Phys. Rev. Research, 1:033190 